\title{
Sodium Currents Activate without a Hodgkin and Huxley- Type Delay in Central Mammalian Neurons
}

\author{
Gytis Baranauskas ${ }^{1}$ and Marco Martina ${ }^{2,3}$ \\ ${ }^{1}$ Psychiatric Institute, University of Illinois at Chicago, Chicago, Illinois 60612 and ${ }^{2}$ Department of Physiology, Feinberg School of Medicine, and ${ }^{3}$ Institute \\ for Neuroscience, Northwestern University, Chicago, Illinois 60611
}

\begin{abstract}
Hodgkin and Huxley established that sodium currents in the squid giant axons activate after a delay, which is explained by the model of a channel with three identical independent gates that all have to open before the channel can pass current (the HH model). It is assumed that this model can adequately describe the sodium current activation time course in all mammalian central neurons, although there is no experimental evidence to support such a conjecture. We performed high temporal resolution studies of sodium currents gating in three types of central neurons. The results show that, within the tested voltage range from -55 to $-35 \mathrm{mV}$, in all of these neurons, the activation time course of the current could be fit, after a brief delay, with a monoexponential function. The duration of delay from the start of the voltage command to the start of the extrapolated monoexponential fit was much smaller than predicted by the HH model. For example, in prefrontal cortex pyramidal neurons, at $-46 \mathrm{mV}$ and $12^{\circ} \mathrm{C}$, the observed average delay was $140 \mu$ sersus the $740 \mu$ s predicted by the two-gate $\mathrm{HH}$ model and the $1180 \mu$ s predicted by the three-gate HH model. These results can be explained by a model with two closed states and one open state. In this model, the transition between two closed states is approximately five times faster than the transition between the second closed state and the open state. This model captures all major properties of the sodium current activation. In addition, the proposed model reproduces the observed action potential shape more accurately than the traditional HH model.
\end{abstract}

Key words: dentate gyrus; granule cell; pyramidal cell; hippocampus; prefrontal cortex; sodium channel; CA1; action potential; activation; kinetics

\section{Introduction}

The classical description of sodium currents is based on the experiments performed on the squid giant axon. Hodgkin and Huxley (1952) showed that, in this preparation, sodium currents activate after a delay. Because a simple two-state model predicts a monoexponential time course of current activation without any delay, they proposed a gating model with three identical, independent gates (the $\mathrm{HH}$ model). According to this model, all of the gates have to open before the channel can pass current; thus, the time required for the three gates to open is responsible for the delay between the voltage command and the beginning of the current flow. The resulting activation time course of sodium currents is best described by the cube of an exponential function (the $m^{3}$ function).

Subsequent studies in large peripheral axons confirmed that sodium currents activate after a delay (Goldman and Schauf, 1973; Keynes and Rojas, 1976; Neumcke et al., 1976; Neumcke and Stampfli, 1982). In all of these experiments, the time course of sodium current activation was best fit with the $m^{n}$ function in

Received June 4, 2005; revised Nov. 28, 2005; accepted Nov. 29, 2005.

This work was in part supported by a University of Illinois at Chicago-Campus Research Board Spring 2003 award (G.B.) and an Epilepsy Foundation Award (M.M.). We thank Bruce Bean and Svirskis Gytis for helpful comments on previous versions of this manuscript.

Correspondence should be addressed to Gytis Baranauskas at his present address: Department of Electronics and Information, Politecnico di Milano, Via Golgi 34/5, 20133 Milano, Italy. E-mail: baranauskas@elet.polimi.it. DOI:10.1523/JNEUROSCI.2283-05.2006

Copyright $\odot 2006$ Society for Neuroscience $\quad$ 0270-6474/06/260671-14\$15.00/0 which $n$ varied from 2 to 4 , implying the presence of two to four independent identical gates. More complex models are required to fully explain all experimental data (French and Horn, 1983; Patlak, 1991), but, because of its simplicity, the $m^{n}$ function (with $n$ equal to 2-4) is universally used to describe the activation time course of sodium currents in all neuronal compartments of all central neurons (Traub and Milles, 1991; Mainen et al., 1995; Martina and Jonas, 1997).

Surprisingly, there is no direct evidence that sodium currents activate after a delay in neurons of the mammalian CNS. The lack of such data can be attributed in part to the insufficient speed of the voltage clamp attained during the whole-cell recordings from the soma of central neurons. Most recordings fail to obtain reliable data in the first $0.2 \mathrm{~ms}$ after the start of voltage step (Mainen et al., 1995; Sherman et al., 1999), which is the expected delay at room temperature (Mainen et al., 1995; Martina and Jonas, 1997) (see Appendix). Therefore, the delay is usually beyond the normal time resolution of voltage-clamp recordings.

Here we report that, in the prefrontal cortex, neurons cooled to $12-14^{\circ} \mathrm{C}$, current whole-cell techniques allow the determination of the sodium current activation kinetics. The actual time resolution of these voltage-clamp recordings was verified by tail current analysis (see Fig. 1). Surprisingly, within the tested voltage range ( -55 to $-35 \mathrm{mV}$ ), sodium currents activated without the $m^{n}$ type delay $(n \geq 2)$. Because this finding contradicts the current consensus about sodium current activation kinetics, we performed nucleated patch recordings from two additional cen- 
tral neuronal types. In these cells, at room temperature, the measured delay of sodium current activation was approximately more than twofold shorter than what is predicted by the $\mathrm{m}^{2}$ function.

We conclude that, in mammalian central neurons, sodium currents activate without a Hodgkin and Huxley-type delay. In addition, we discuss the consequence of such results on the electrophysiology of central neurons.

\section{Materials and Methods}

Electrophysiology. The data presented in this paper were obtained from experiments performed using three types of electrophysiological techniques: whole-cell recordings of dissociated prefrontal cortex neurons, whole-cell recordings from prefrontal cortex slices, and nucleated patch recordings of granule cells of the dentate gyrus and CA1 pyramidal cells.

For prefrontal cortex electrophysiology experiments, acute brain slices were obtained from 4- to 7-week-old rats following a standard protocol described previously (Baranauskas, 2004). All procedures were reviewed by the University of Illinois at Chicago Animal Care Committee and met the requirements outlined in the National Institutes of Health Guide for the Care and Use of Laboratory Animals. In brief, rats were anesthetized with sodium pentobarbital $(50 \mathrm{mg} / \mathrm{kg}$ ) and decapitated. The brains were rapidly removed, and the frontal part of the forebrain was sliced into 400 - $\mu \mathrm{m}$-thick coronal slices (cut at a slightly oblique angle of $\sim 30^{\circ}$ ) in ice-cold oxygenated solution consisting of the following (in $\mathrm{mM}$ ): 100 $\mathrm{NaCl}, 2.5 \mathrm{KCl}, 2 \mathrm{MgCl}_{2}, 15 \mathrm{HEPES}, 1 \mathrm{CaCl}_{2}, 1 \mathrm{Na}_{2} \mathrm{HPO}_{4}, 15$ glucose, and $26 \mathrm{NaHCO}_{3}$, pH 7.35 with $\mathrm{NaOH}$ (osmolarity, 295-305 mOsm/L). The slices were then placed in the same solution with added $3 \mathrm{~mm}$ sodium pyruvate and $1 \mathrm{~mm}$ ascorbic acid and kept at $36^{\circ} \mathrm{C}$.

For current-clamp recordings in prefrontal cortex slices, the internal solution consisted of the following (in $\mathrm{mM}$ ): $90 \mathrm{~K}_{2} \mathrm{SO}_{4}, 2 \mathrm{MgCl}_{2}, 30$ HEPES, 0.5 EGTA, and $5 \mathrm{Na}_{2} \mathrm{ATP}$, pH 7.2 with $\sim 15 \mathrm{NaOH}$ (osmolarity, $260-270 \mathrm{mOsm} / \mathrm{L}$ ). The external solution was the same as the one used to prepare slices. All recordings in slices were performed at room temperature $\left(22-24^{\circ} \mathrm{C}\right)$.

Pyramidal neurons from the dorsal part of the prelimbic area (Groenewegen and Uylings, 2000) were selected according to their shape and the presence of a long apical dendrite. Staining with Lucifer yellow confirmed the presence of long apical dendrite, reaching the pial surface of the slice ( $n=4$; data not shown).

For acutely dissociated cells, the internal solution consisted of the following (in mM): $75 \mathrm{~K}_{2} \mathrm{SO}_{4}, 2 \mathrm{MgCl}_{2}, 30$ HEPES, 10 EGTA, and 5 $\mathrm{Na}_{2} \mathrm{ATP}$ or $5 \mathrm{~K}_{2} \mathrm{ATP}$, pH 7.2 with $\sim 15 \mathrm{NaOH}$ (osmolarity, 260-270 $\mathrm{mOsm} / \mathrm{L}$ ). The external solution consisted of the following (in $\mathrm{mm}$ ): 2 $\mathrm{BaCl}_{2}, 30 \mathrm{HEPES}, 110 \mathrm{CsCl}, 0.1 \mathrm{CdCl}_{2}, 10 \mathrm{NaCl}$, and 20 tetraethylammonium (TEA)-Cl. Osmolarity was $300-310 \mathrm{mOsm} / \mathrm{L}$, $\mathrm{pH}$ adjusted to 7.35 with $\mathrm{CsOH}$. Solutions were applied by a gravity-fed sewer pipe system.

Prefrontal cortex pyramidal neurons were visually identified as large pyramidal-shaped cells. In five cells, the neuron type was later confirmed by detection of calcium/calmodulin-dependent protein kinase II (CaMKII) mRNA and the absence of the $67 \mathrm{kDa}$ isoform of glutamate decarboxylase $\left(\mathrm{GAD}_{67}\right)$ mRNA (see Fig. $1 \mathrm{~A}$ and below, Single-cell RT-PCR).

Most of the recordings were performed at $12-14^{\circ} \mathrm{C}$ using the Dagan (Minneapolis, MN) TC100 temperature controller. The remaining experiments were performed at room temperature $\left(22-24^{\circ} \mathrm{C}\right)$. Recordings were obtained with a Dagan 3900 patch-clamp amplifier and controlled and monitored with personal computer running pClamp software (version 8.0) (Axon Instruments, Union City, CA). In working solution, electrode resistance was 1.5-3.0 M $\Omega$. Electrodes were coated with Semiconductor Protective Coating (Dow Corning, Midland, MI). After seal rupture, series resistance (3.4-8.5 $\mathrm{M} \Omega$ ) was compensated (50-80\%) and monitored periodically. A supercharge technique (Armstrong and Chow, 1987) was used to minimize the time required for the membrane charging after the voltage step. This time was always $\leq 150 \mu$ s for voltage steps of $<30 \mathrm{mV}$. A series of tests were used to verify the quality and speed of the voltage clamp (see Fig. 1 and Results). Voltage-clamp recordings were low-pass filtered at a cutoff frequency of $20 \mathrm{kHz}$ using an eight-pole filter. Current traces were leak subtracted using the following procedures. For tail currents, the sodium current was first inactivated by a 50 $\mathrm{ms}$ voltage step to $-36 \mathrm{mV}$ and then followed by a tail current protocol as shown in Figure $1 B$ (without returning to $-76 \mathrm{mV}$ ). For $I-V$ measurements, leak currents were estimated by stepping from -85 to -80 to -55 $\mathrm{mV}$.

For nucleated patch recordings from dentate gyrus granule cells and CA1 pyramidal cells, 16-to 18-d-old rats were anesthetized with isoflurane and killed by decapitation in accordance with national and institutional guidelines. Transverse $300-\mu \mathrm{m}$-thick hippocampal slices were cut using a vibratome (Dosaka, Kyoto, Japan). Granule cells of the dentate gyrus and CA1 pyramidal cells were identified visually by infrared differential interference contrast video microscopy (Stuart and Sakmann, 1994) using an upright microscope (Zeiss, Oberkochen, Germany) equipped with a $60 \times$ water immersion objective (Olympus Optical, Tokyo, Japan) and by their characteristic action potential pattern during sustained current injection.

Patch pipettes were pulled from borosilicate glass tubing ( $2 \mathrm{~mm}$ outer diameter, $0.5 \mathrm{~mm}$ wall thickness; Hilgenberg, Malsfeld, Germany), fire polished immediately before use, and coated with thin stripes of Parafilm. Neurons were approached under visual control while applying positive pressure $(20-40 \mathrm{mmHg})$ to the pipettes. The pipette resistance (in working solution) was 2-5 M $\Omega$, and the series resistance in the wholecell configuration ranged from 4 to $14 \mathrm{M} \Omega$. Only neurons with initial membrane potentials negative to $-60 \mathrm{mV}$ were accepted for recording. To isolate nucleated patches (Martina and Jonas, 1997), negative pressure $(40-100 \mathrm{mmHg})$ was applied, and the pipette was withdrawn slowly under visual control. Nucleated patches had input resistance $\geq 2 \mathrm{G} \Omega$, almost spherical shape, and diameter of $5-9 \mu \mathrm{m}$. A light negative pressure $(3-5 \mathrm{mmHg}$ ) was maintained during the recordings.

Currents were recorded using an Axopatch 200 B amplifier (Axon Instruments). Signals were filtered at $100 \mathrm{kHz}$ using the built-in low-pass filter. Capacitive transients were minimized by maintaining the bath level as low as possible and further reduced by the capacitive compensation circuit of the amplifier. A Digidata 1300 interface connected to a personal computer was used for stimulus generation and data acquisition. The sampling frequency was $200 \mathrm{kHz}$. Nucleated patches were held at -90 $\mathrm{mV}$, and a pulse to $-120 \mathrm{mV}$ was applied before each test pulse to obtain complete recovery from fast inactivation.

Potassium currents were blocked by internal cesium and calcium current by external $\mathrm{CdCl}_{2}(50 \mu \mathrm{M})$. During recording, slices were continuously superfused with physiological extracellular solution containing the following (in mM): $125 \mathrm{NaCl}, 25 \mathrm{NaHCO}_{3}, 2.5 \mathrm{KCl}, 1.25 \mathrm{NaH}_{2} \mathrm{PO}_{4}, 1.2$ $\mathrm{CaCl}_{2}, 1 \mathrm{MgCl}_{2}$, and 25 glucose (bubbled with $95 \% \mathrm{O}_{2}$ and $5 \% \mathrm{CO}_{2}$ ). Pipette's internal solution contained the following (in $\mathrm{mM}$ ): $140 \mathrm{CsCl}, 2$ $\mathrm{MgCl}_{2}, 10$ EGTA, $2 \mathrm{Na}_{2}$ ATP, $0.3 \mathrm{GTP}$, and 10 HEPES, pH adjusted to 7.3 with $\mathrm{KOH}$. In some experiments, $10 \mathrm{~mm}$ TEA was added to the external solution. Because no differences were noticed in the currents at the membrane potential values tested, data were pooled. In five cells, the currents were completely blocked by the application of 500 nм TTX-containing solution, confirming that the observed currents were mediated by the fast TTX-sensitive sodium channels. Leakage and capacitive currents were subtracted online using a $\mathrm{P} /-5$ protocol.

The liquid junction potential was measured according to the method described by Neher (1992) and by calculating with the pClamp tool. The liquid junction potential was $6 \mathrm{mV}$ for acutely dissociated cells, $9 \mathrm{mV}$ for the recordings in slices, and $4 \mathrm{mV}$ for nucleated patches. These values were subtracted from the command voltages off-line, and the corrected values are used in this paper.

All data analysis was performed with Igor Pro (WaveMetrics, Lake Oswego, OR). All averaged data are represented as an average \pm SEM. Traces in the figures were filtered digitally $(5-20 \mathrm{kHz})$ and represent the average of up to 20 sweeps. Permeability voltage dependence was obtained from the corrected peak current-voltage relationship using the Goldman-Hodgkin-Katz equation (Hille, 2001). Peak current amplitude correction was performed by extrapolating the exponential part of the current decay to the start of the test voltage command. Inactivation voltage dependence was measured with 500-ms-long conditioning steps. 
All drugs were obtained from Sigma (St. Louis, MO), except for tetrodotoxin, which was obtained from Alomone Labs (Jerusalem, Israel).

Single-cell reverse transcription-PCR. The reverse transcription (RT)PCR procedure and the primers used have been described previously (Tkatch et al., 1998; Vysokanov and Flores-Hernandez, 1998; Baranauskas, 2004).

Briefly, neurons were recorded in a whole-cell mode with a minimal volume of internal solution $(<3 \mu \mathrm{l}$; see above) and aspirated into the electrode for $\mathrm{GAD}_{67}$ and CaMKII mRNA detection. The RT was performed using the SUPERSCRIPT First-Strand Synthesis System (Invitrogen, Carlsbad, CA). All RT-PCR runs included negative and positive controls. Contamination from extraneous sources was checked by performing the RT-PCR procedure in exactly the same way as described above, except that no pipette content was expelled into the RT tube. The contribution of genomic DNA was checked by omission of the RT reaction. None of these negative controls resulted in a band on the gel. The cell-harvesting process was visually controlled, and cells were not used if any additional debris entered the pipette. Highly diluted tissue cDNA was used as a positive control for PCR reaction.

Computer simulations. We constructed a six-state sodium current model (see Fig. $5 A$ ). The transition rates from the second closed state to the open state $(\alpha 2$ and $\beta 2)$ and the transition rates to the inactivated states $(\alpha 3$ and $\beta 3)$ were obtained from the fits shown in Figures 3, $A$ and $B$, and $4 B$. It was assumed that all of these rates saturate except $\beta 3$. The following functions describe the voltage dependence of these rate constants: $\alpha 2=2.8^{((\mathrm{T}-13) / 10)} \times 11 /(0.4+\exp (-(V+6) / 12)) ; \beta 2=$ $2.8^{((\mathrm{T}-13) / 10)} \times 0.035 /(0.0015+\exp ((V+6) / 12)) ; \alpha 3=2.4^{((\mathrm{T}-13) / 10)} \times$ $2 /(2+\exp (-(V+6) / 12)) ;$ and $\beta 3=2.4^{((\mathrm{T}-13) / 10)} \times 0.00005 \times$ $\exp (-(V+6) / 13)$. Here $\mathrm{T}$ is temperature in degrees Celsius, and $V$ is membrane voltage in millivolts. The units of the rate constants are milliseconds.

The transition from the first to the second closed state helps to account for the observed steep voltage dependence of the sodium current amplitude and the presence of very brief delay $\left(<0.2 \mathrm{~ms}\right.$ at $12^{\circ} \mathrm{C}$; see Results). Therefore, $\alpha 1$ was adjusted to reproduce the current amplitudes observed during voltage-clamp recordings at less than $-40 \mathrm{mV}$ while maintaining the slowest time constant of this transition $<0.2 \mathrm{~ms}$. The following rates were used in the model: $\alpha 1=2.8^{((\mathrm{T}-13) / 10)} \times 10 \times \exp ((V+$ $6) / 45)$; and $\beta 1=2.8^{((\mathrm{T}-13) / 10)} \times 0.35 \times \exp (-(V+6) / 8)$.

Because in this model, the kinetics of sodium current activation is primarily determined by a single transition from the second closed state to the open state, we will refer to this model as the $m^{1}$ sodium current model. For comparison, a widely used HH model with three gates (Mainen et al., 1995) will be referred to as the $m^{3}$ sodium current model.

All computations were performed using the simulation program NEURON (Hines, 1993). The fully implicit backward Euler integration method was used with a time step of $25 \mu$ s.

Voltage-clamp traces were simulated in a patch configuration assuming an internal sodium concentration of $34 \mathrm{~mm}$ and external sodium concentration of $10 \mathrm{~mm}$. Current-clamp simulations used a model of pyramidal cell that is available with the demonstration files of the program. The soma size was reduced to $32 \times 19 \mu \mathrm{m}^{2}$. The obtained soma surface area $\left(32 \times 19 \times 3.14=1909 \mu \mathrm{m}^{2}\right)$ is close to the surface area estimated from the average capacity of $14 \mathrm{pF}(n=36)$ obtained during recordings in slices (assuming $0.75 \mu \mathrm{F} / \mathrm{cm}^{2}, 14 \mathrm{pF}$ corresponds to 1867 $\mu \mathrm{m}^{2}$ ). Sodium currents were calculated according to the GoldmanHodgkin-Katz equation, whereas potassium and leak currents were described using a classical Ohm law. This approach is justified by the fact that other authors reported their data in the terms of conductance densities.

Two types of models were tested. In the first type of model, we assumed that the sodium channel density in the axonal and somatic compartments were the same. In the second type of model, the sodium channel density in the axonal compartment was approximately eight times higher than in the soma (Safronov et al., 2000). In this case, we were able to reproduce an asymmetrical time course of the depolarization speed (see Fig. $5 I, J)$. Both types of models were used for simulations with the $m^{1}$ sodium channel model and the $m^{3}$ sodium channel model. Thus, in total, the results of four current-clamp simulations are reported in this paper.
The following parameters were used during simulations incorporating the $m^{1}$ sodium current model.

In the case of the first type of model, the axonic compartment included the sodium current (permeability, $3.2 \times 10^{-4} \mathrm{~cm} / \mathrm{s}$ ), a slow potassium current $\left(18 \mathrm{mS} / \mathrm{cm}^{2}\right)$ (Korngreen and Sakmann, 2000) (model provided by Migliore et al., 2001), a delayed rectifier current $\left(30 \mathrm{mS} / \mathrm{cm}^{2}\right)$ (Durstewitz et al., 2000), and the leak current $\left(0.02 \mathrm{mS} / \mathrm{cm}^{2}\right)$. The somatic compartment contained the sodium current (permeability, $3.2 \times 10^{-4}$ $\mathrm{cm} / \mathrm{s})$, the slow potassium current $\left(12 \mathrm{mS} / \mathrm{cm}^{2}\right)$, a fast potassium current $\left(1 \mathrm{mS} / \mathrm{cm}^{2}\right.$ ) (Korngreen and Sakmann 2000) (model provided by Migliore et al., 2001), and the leak current $\left(0.02 \mathrm{mS} / \mathrm{cm}^{2}\right)$. In the soma compartment, the used sodium channel permeability was within the range of the observed permeability values in prefrontal cortex pyramidal neurons. During voltage-clamp experiments in acutely dissociated cell, on average, the observed sodium channel permeability was $2.5 \pm 0.2 \times$ $10^{-4} \mathrm{~cm} / \mathrm{s}\left(n=8\right.$; range from $1.2 \times 10^{-4}$ to $\left.3.9 \times 10^{-4} \mathrm{~cm} / \mathrm{s}\right)$. This permeability value corresponds to the $\sim 60 \mathrm{mS} / \mathrm{cm}^{2}$ conductance density measured at $-50 \mathrm{mV}$ (assuming the reversal potential of $+57 \mathrm{mV}$ ) that is $\sim 10$ times higher than the one reported by several groups (Stuart and Sakmann, 1994; Martina and Jonas, 1997). One possible explanation of this difference is the use of adult animals ( $>6$ weeks old) for the current study as opposed to the 2 -week-old rats used by other groups. Alternatively, in dissociated cells, channel density is higher than in nucleated patches or cell-attached patches because of technical differences.

All dendritic branches contained the leak conductance $(0.025 \mathrm{mS} /$ $\mathrm{cm}^{2}$ ). In addition, the first $300 \mu \mathrm{m}$ of the apical dendrite contained sodium current (decreasing from $3.1 \times 10^{-4}$ to $2.3 \times 10^{-4} \mathrm{~cm} / \mathrm{s}$ ), slow potassium current (decreasing from 12 to $8 \mathrm{mS} / \mathrm{cm}^{2}$ ), and fast potassium current $\left(1 \mathrm{mS} / \mathrm{cm}^{2}\right)$. It has been reported that, in cortical pyramidal neurons, the density of sodium and potassium channels slowly decreases along the apical dendrite (Huguenard et al., 1989; Stuart and Sakmann, 1994; Korngreen and Sakmann, 2000). It has been assumed that similar distribution is valid for prefrontal cortex neurons. Dendritic sodium currents helped to account for the observed amplitude of the action potential. The leak conductance value was adjusted so that a small current step (10 pA) would evoke membrane depolarization similar to the observed depolarization in a real neuron.

The reversal potential of sodium current was set to $57 \mathrm{mV}$. The external potassium concentration was set to $3 \mathrm{~mm}$ and the internal potassium concentration was $180 \mathrm{~mm}$, in agreement with the composition of solutions used for the slice recordings. The slow potassium current density was adjusted to obtain a spike width at $-30 \mathrm{mV}$, similar to the one experimentally recorded in slices.

In the case of the second type of model, the axonic compartment included the sodium current $\left(19 \times 10^{-4} \mathrm{~cm} / \mathrm{s}\right)$, the slow potassium current $\left(11 \mathrm{mS} / \mathrm{cm}^{2}\right)$, the delayed rectifier current $\left(80 \mathrm{mS} / \mathrm{cm}^{2}\right)$, and the leak current $\left(0.02 \mathrm{mS} / \mathrm{cm}^{2}\right)$. The somatic compartment contained the sodium current $\left(2.5 \times 10^{-4} \mathrm{~cm} / \mathrm{s}\right)$, the slow potassium current $(11 \mathrm{mS} /$ $\left.\mathrm{cm}^{2}\right)$, the fast potassium current $\left(0.5 \mathrm{mS} / \mu \mathrm{m}^{2}\right)$, and the leak current $\left(0.02 \mathrm{mS} / \mathrm{cm}^{2}\right)$. Current densities in dendrites were scaled in the same manner as in the first model.

In the case of high axonal sodium channel density, the observed action potential threshold could be obtained only after the sodium current halfactivation voltage was shifted by $6 \mathrm{mV}$ with respect to the data obtained from the dissociated cells. This shift may be attributable to differences in the phosphorylation state of the channels in dissociated cells compared with slices. Phosphorylation is known to affect the voltage dependence of sodium channels (Catterall, 2000). The second explanation can be associated with inaccurate description of potassium currents that were taken from the motor-cortex pyramidal neuron and may not be suitable for the prefrontal cortex neurons. Thus, the contribution of potassium current to the setting of the spike threshold was not appropriate. Without the voltage shift, the shape of action potential was very similar to the recorded spike shape, with the only difference being the threshold of the action potential.

The following parameters were used during simulations incorporating the $m^{3}$ sodium current model.

In the case of the second type of model, all conductance densities were the same as in the case of simulations with the $m^{1}$ model with the same 
sodium channel density in the axonal and somatic compartments, except that the sodium current permeability and the conductance density of the slow potassium currents was increased by $10 \%$. In these simulations, to obtain the observed threshold of the action potential, the voltage dependence of the sodium current was shifted by $-1 \mathrm{mV}$.

In the case of the second type of model, the axonic compartment included the sodium current $\left(2.4 \times 10^{-4} \mathrm{~cm} / \mathrm{s}\right)$, the slow potassium current $\left(15 \mathrm{mS} / \mathrm{cm}^{2}\right)$, the delayed rectifier current $\left(150 \mathrm{mS} / \mathrm{cm}^{2}\right)$, and leak current $\left(0.02 \mathrm{mS} / \mathrm{cm}^{2}\right)$. The somatic compartment contained the sodium current (permeability, $3.6 \times 10^{-4} \mathrm{~cm} / \mathrm{s}$ ), slow potassium current $\left(15 \mathrm{mS} / \mathrm{cm}^{2}\right)$, the fast potassium current $\left(0.5 \mathrm{mS} / \mathrm{cm}^{2}\right)$, and a leak current $\left(0.02 \mathrm{mS} / \mathrm{cm}^{2}\right)$. In both cases, the drop-off of dendritic sodium and slow potassium channel densities was qualitatively similar the one used in the simulations with the $m^{1}$ sodium channel model. In these simulations, to obtain the observed threshold of the action potential, the voltage dependence of the sodium current was shifted by $7 \mathrm{mV}$.

\section{Results}

Currents recorded at $12^{\circ} \mathrm{C}$ had properties of typical fast sodium currents

Electrically compact neurons were obtained by acute dissociation of rat prefrontal cortex. Cells with pyramidal shape and a short $(<20 \mu \mathrm{m})$ stump of the apical dendrite were selected for the recordings. In these neurons, the TTX-sensitive currents recorded at $12^{\circ} \mathrm{C}$ activated very rapidly $(<2 \mathrm{~ms})$ and then decayed with time constants ranging from $\sim 1$ to $\sim 35 \mathrm{~ms}$ (Fig. 1A). Hence, the recorded current was similar to the fast TTX-sensitive sodium current described in prefrontal cortex pyramidal neurons (Maurice et al., 2001).

Because several types of neurons are found in the layer $\mathrm{V}$ of the rat frontal cortex (Kawaguchi, 1993), we used single-cell RT-PCR to characterize the cell type in a subset of the recorded neurons $(n=5)$. In these neurons, cell identification was performed by testing the presence of the CaMKII and $\mathrm{GAD}_{67}$ mRNAs. As shown in Figure $1 A$ (inset), all neurons were $\mathrm{GAD}_{67}$ negative but positive for CaMKII, demonstrating that all of these neurons were glutamatergic. All five neurons satisfied the below described requirements for the voltage-clamp speed and were used for the sodium current analysis.

\section{Voltage-clamp quality was sufficient to determine the} activation time course of sodium currents

Because the goal of these experiments was to establish the activation kinetics of sodium currents, it was critical to verify the quality of voltage clamp in our experimental conditions. To this aim, tail currents representing the deactivation kinetics were recorded (Fig. $1 B, C$ ). At constant voltage, the time course of sodium channel closure can be usually fit with a monoexponential function (Hodgkin and Huxley, 1952). Because the time constant of the monoexponential function is voltage sensitive, any loss of voltage control will result in a non-monoexponential decay of the current. In addition, suboptimal voltage control will translate into unstable driving force for sodium ions, thus affecting the amplitude of the sodium current and additionally deteriorating the monoexponential time course of the current decay. The current time course matched very closely the monoexponential fit function after $\sim 120 \mu$ s from the start of the voltage step (Fig. 1D). Hence, there is a reasonable voltage control of the current after $\sim 120 \mu$ s from the start of the voltage command for voltage steps of $<30 \mathrm{mV}$.

An additional control for the speed and quality of the voltage clamp can be made by plotting the peak amplitude of the tail current against the voltage of the command step. In this case, the fraction of open channels at the beginning of the voltage com-

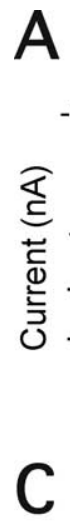

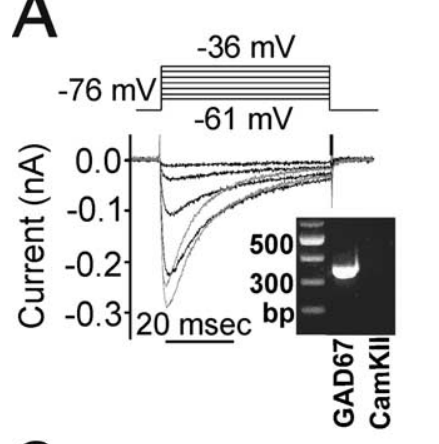

B
$-76 \mathrm{mV} \sqrt{\frac{-36 \mathrm{mV}}{-56 \mathrm{mV}}}$

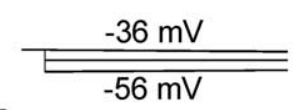

D

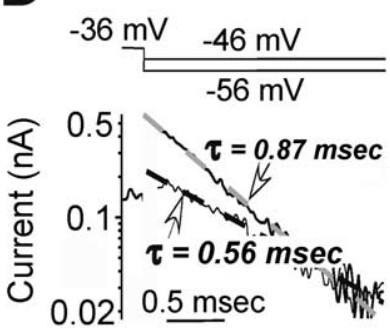

$E$

$\mathbf{F}$
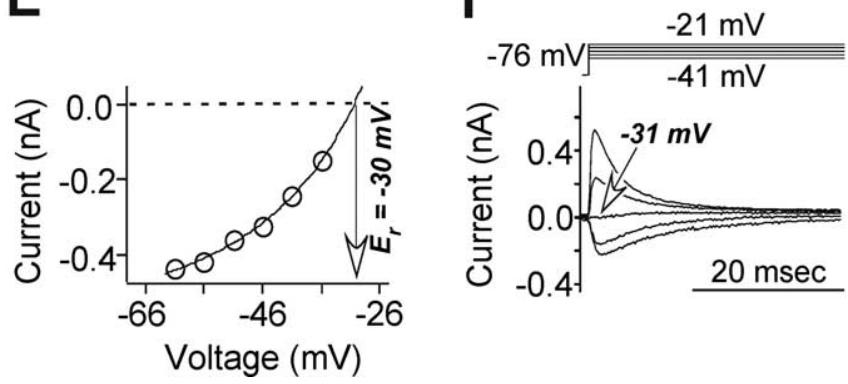

Figure 1. The speed of voltage clamp in acutely dissociated prefrontal cortex neurons is sufficient to investigate activation kinetics of sodium current at $12^{\circ} \mathrm{C} . A$, An example of current traces evoked by the voltage-clamp protocol shown above the traces. The inset shows a gel obtained after the RT-PCR analysis of the recorded neuron, and its current traces are shown in $\boldsymbol{A}-\boldsymbol{D}$ and $\boldsymbol{F}$. CaMKII mRNA but not $\mathrm{GAD}_{67}$ mRNA was detected in this neuron. $\boldsymbol{B}$, The voltageclamp protocol shown above the traces was used to study the deactivation kinetics of sodium currents (tail currents). The dark thick trace corresponds to the $-56 \mathrm{mV}$ voltage step, the dark thin trace corresponds to the $-46 \mathrm{mV}$ voltage step, and the light thick trace corresponds to the $-36 \mathrm{mV}$ voltage step. $\boldsymbol{C}$, The same traces of $\boldsymbol{B}$ are shown on an expanded time scale. Note the increase in the peak amplitude of the tail current at more hyperpolarized membrane potentials. The first $\sim 100 \mu$ s after the start of the test voltage step were blanked for clarity. D, A semilogarithmic plot of the fast component of the traces shown in $\mathbf{C}$ demonstrates the monoexponential decay of the tail currents starting $\sim 100 \mu$ s after the start of the voltage step. The slow component of the traces was subtracted as shown in Figure $2 G$. Dashed lines represent the monoexponential fits. $\boldsymbol{E}$, Total amplitude of the tail current measured at the start of the test step (120-220 $\mu$ s after the start of the test pulse) plotted versus the voltage of the test step. The thin line represents the Goldman-Hodgkin-Katz equation fit of the data points. The arrow points to the extrapolated reversal potential of the tail currents $(-30.5 \mathrm{mV})$. $\boldsymbol{F}$, The reversal potential of approximately $-30 \mathrm{mV}$ was obtained by direct measurement. All traces are from the same neuron and were obtained by subtracting the currents obtained in the presence of 0.5 $\mu \mathrm{M}$ TTX from the control traces. All traces were digitally filtered for clarity ( $2 \mathrm{kHz}$ for $\boldsymbol{A}, \boldsymbol{B}, \boldsymbol{F} ; 10$ $\mathrm{kHz}$ for $\boldsymbol{C}, \boldsymbol{D})$. Traces in $\boldsymbol{A}-\boldsymbol{D}$ are the averages of four sweeps.

mand is the same for all test voltages, and the peak current amplitude must be proportional to the driving force for sodium ions. Figure 1, $E$ and $F$, shows that the sodium ion reversal potential estimated from the tail currents was similar to the reversal potential obtained from the peak current measurements in the same cell. The value of the measured reversal potential for the sodium current was close to the calculated reversal potential of 
$-22 \mathrm{mV}$ for the solutions used during these recordings ( $10 \mathrm{mM}$ $\mathrm{Na}^{+}$in the external solution and $25 \mathrm{mM}$ of $\mathrm{Na}^{+}$in the pipette solution; see Materials and Methods).

Similar analysis indicated that an acceptable voltage-clamp control was achieved after $\leq 150 \mu \mathrm{s}$ in nine cells recorded at $12-14^{\circ} \mathrm{C}$ (including five cells used for RT-PCR) and seven cells recorded at room temperature $\left(22-24^{\circ} \mathrm{C}\right)$. Only these cells were further analyzed.

The time resolution of the currents $(\sim 150 \mu \mathrm{s})$ was sufficient to detect the delay of currents activation when the activation time constants are $>0.5 \mathrm{~ms}$ (for details, see Appendix).

\section{Sodium currents activate without the $m^{n}$ type delay at $12^{\circ} \mathrm{C}$ in prefrontal cortex neurons}

For traces obtained at $12^{\circ} \mathrm{C}$, a preliminary examination of the sodium current activation revealed no delay at less than $-40 \mathrm{mV}$ (Fig. 2A). However, a more detailed examination of the time course of the sodium current activation can be performed by the mathematical elimination of inactivation (Keynes and Rojas, 1976). In this procedure, the current trace is treated as a multiple of two components: the raising phase or "activation" component $1-\exp \left(-t / \tau_{\text {act }}\right)^{n}$, where $n=1-3$, and the decaying phase or "inactivation" component $\exp \left(-t / \tau_{\text {inact }}\right)$. By dividing the current trace by the decaying component $\exp \left(-t / \tau_{\text {inact }}\right)$, the raising phase or activation component $1-\exp \left(-t / \tau_{\text {act }}\right)$ is revealed as a nondecaying trace (Fig. $2 D$, inset) (for details, see Appendix). Hence, following this procedure, the time course of current raising phase should be determined solely by the function of $\exp \left(-t / \tau_{\text {act }}\right)$.

It should be noted that, for such analysis, we assume that macroscopic inactivation time constants correspond to the microscopic inactivation time constants and that inactivation and activation processes are independent. Single-channel recordings have shown that macroscopic inactivation rates may not correspond to the microscopic inactivation rates (Aldrich et al., 1983); since then, the sodium channel inactivation process is usually assumed to be either voltage independent (Kuo and Bean, 1994) or little voltage dependent (Vandenberg and Bezanilla, 1991). The relevance of these models to the data presented here is discussed in the section of the model selection for computer simulations. Nevertheless, computer simulations show that, in most of the cases, the mathematical procedure used in this study "to remove" inactivation is suitable to reveal the kinetics of the step(s) controlling activation process. For instance, in the case of the sequential model "closed state" $\leftrightarrow$ "open state" $\leftrightarrow$ "inactivated state" in which the first transition is slow whereas the second is fast (Hille, 2001), this procedure will identify the second transition time constant, the one that determines the activation kinetics in this model. Moreover, for voltage steps to less than $-40 \mathrm{mV}$, the correction is minimal and unlikely to introduce any artifacts.

Nevertheless, we realize that it is a purely mathematical procedure, and caution should be used while applying the procedure. Hence, we used a double-pulse protocol to detect possible presence of a rapid inactivation that may interfere with the activation process (Hille, 2001). Figure $2 C$ shows that there was a good match between the current decay after a step to $-46 \mathrm{mV}$ and the estimated inactivation time course measured with a protocol shown in Figure $2 B$. Importantly, there is no additional rapid decay phase during the first $2 \mathrm{~ms}$ after the start of the test command. In both cases, the time course could be fit with a monoexponential function, and the obtained time constants differed by $<30 \%$. This difference may be attributable to the relatively brief duration of the test command (two to three times that of the inactivation time constant).
A

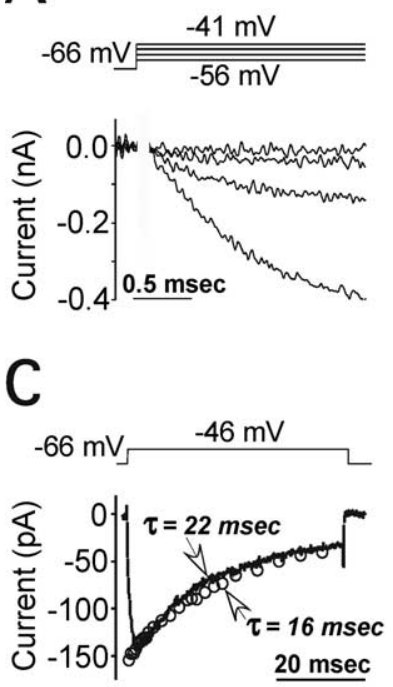

$E$
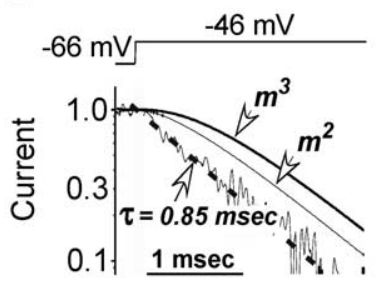

G

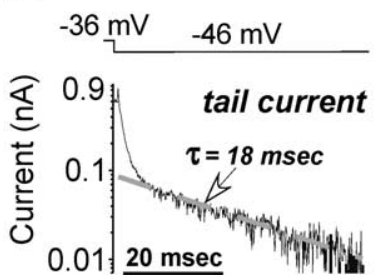

B

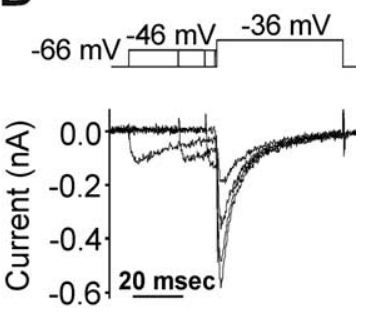

D

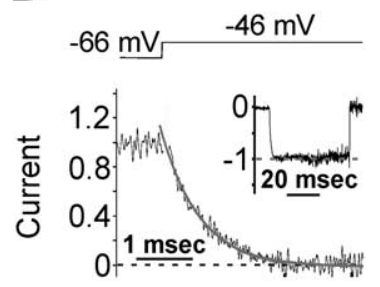

$\mathbf{F}$

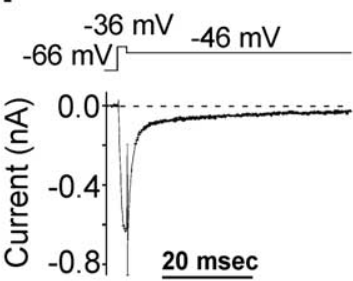

H

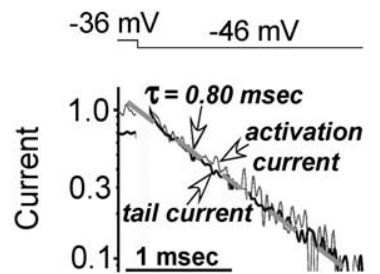

Figure 2. Sodium current activation can be fit with a monoexponential function at $12^{\circ} \mathrm{C}$. $\boldsymbol{A}$, An example of the sodium current activation recorded from an acutely dissociated prefrontal cortex neuron. Currents were evoked by the voltage-clamp protocol shown above the traces. $\boldsymbol{B}$, Voltage clamp protocol used to test the presence of a fast component of inactivation. Currents were evoked by the protocol shown above the traces; the inactivation time course at $-46 \mathrm{mV}$ was estimated by measuring the amplitude of the peak current at $-36 \mathrm{mV}$. C, There was a clear overlap between the inactivation time course obtained by the protocol shown in $\boldsymbol{B}$ and the decay of sodium current evoked with a voltage step to $-46 \mathrm{mV}$. No fast inactivation was detected during the first $2-5 \mathrm{~ms}$. $\boldsymbol{D}$, The inactivation of the current trace shown in $\boldsymbol{C}$ was eliminated mathematically by dividing the current trace by the following function: $28.8 \mathrm{pA}+$ $134.5 \mathrm{pA} \times \exp (-t / 16.1 \mathrm{~ms})$. The inset shows that this procedure converted the decaying current to a nondecaying current. The main panel shows the same converted trace on an expanded time scale and after the subtraction of the trace from 1 . The light line represents the monoexponential fit of the activation. Note the absence of any detectable delay. For details of all procedures, see Appendix. $\boldsymbol{E}$, A semilogarithmic plot of the trace from $\boldsymbol{D}$. The monoexponential fit is shown as a thick dashed line. Two smooth lines correspond to the quadrate (thin line) and the cube (thick line) of an exponential function. $\boldsymbol{F}$, Tail current evoked by the voltage-clamp protocol shown above the traces. $\boldsymbol{G}$, A semilogarithmic plot of the tail current shown in $\boldsymbol{F}$ reveals the presence of two distinct components. The slowly decaying component was fit with a monoexponential function with a time constant of $18 \mathrm{~ms}$, which is close to the inactivation rate of the current at $-46 \mathrm{mV}(\boldsymbol{C})$. This slow component was subtracted from the original trace, and the semilogarithmic plot of the fast component is shown in $\boldsymbol{H}$. $\boldsymbol{H}$, The monoexponential fit for the fast component of the tail current (dark line) is shown as a light dashed line. Note that the obtained rate of tail current decay is similar to the activation rate at this voltage ( $0.80 \mathrm{vs} 0.85 \mathrm{~ms}$; $\boldsymbol{E}$ ). For comparison, the activation current trace from $\boldsymbol{E}$ is shown as a thin light line. 
The elimination of inactivation was performed by dividing the current trace by the monoexponential fit function (see Appendix). The result is a nondecaying trace (Fig. $2 D$, inset). The absence of any significant deviation of the obtained current amplitude from 1 (except during the activation process for the first 2-3 $\mathrm{ms}$ ) confirms that the original current trace decayed as a monoexponential function. The same observation was made for all nine neurons that were analyzed using this method for voltages between -56 and $-40 \mathrm{mV}$. At membrane potentials above -40 $\mathrm{mV}$, a second slower component of inactivation could be detected. However, this second component comprised $<30 \%$ of the total current amplitude and was very slow (inactivation time constants $>20 \mathrm{~ms}$ at $12-14^{\circ} \mathrm{C}$ ). Therefore, during the analysis of the sodium current activation time course, the slower inactivation component was included into the non-inactivating term of the exponential function used to eliminate inactivation (for details, see Appendix).

Figure $2 D$ shows that the current activation time course could be fit with a monoexponential function. Therefore, the current trace obtained in the semilogarithmic plot follows a straight line (Fig. 2E). This linear portion of the trace was used to determine the activation time constant $\tau_{m}$. For comparison, the lines corresponding to the square and the cube of an exponential function with the same time constant $\tau_{m}$ have been added to this plot (Fig. $2 E$ ) (for details, see Appendix). It is obvious that the activation time course is very different from the square or the cube of an exponential function.

The same clear difference between the $m^{2}$ and the $m^{3}$ function and the actual current traces was observed in all nine neurons that satisfied the above described criteria for the quality of the voltage clamp at $12-14^{\circ} \mathrm{C}$. In Figure $2 \mathrm{E}$, the duration of the delay can be measured as the time interval between the start of the voltage command and the time when the extrapolated monoexponential fit function is equal to 1 (Fig. $2 E$ ) (for details, see Appendix). For current traces obtained at the test voltage of $-46 \mathrm{mV}$ (holding potential of -66 or $-76 \mathrm{mV}$ ), the average delay was $140 \mu \mathrm{s}$ $\left(0.13 \pm 0.02 \times \tau_{m}\right.$, ranging between $0.02 \times \tau_{m}$ and $0.19 \times \tau_{m}$, where $\tau_{m}$ is the time constant of the monoexponential fit; $n=9$ ). Similar observations were made for test voltages between -56 and $-36 \mathrm{mV}$. In this range of voltages and for holding potentials of either -76 or $-66 \mathrm{mV}$, the delay never exceeded $270 \mu$ s and was always $<0.3 \times \tau_{m}$. Because the estimated membrane charging time is $<100-150 \mu$ s (Fig. 1 ), such brief delays may be caused at least in part by membrane charging. However, these data do not exclude the existence of a brief delay (see below) similar to what reported for the Kv3 potassium currents (Lien and Jonas, 2003).

For holding potentials of -76 or $-66 \mathrm{mV}$, it is possible that the delay in the activation of the sodium current is reduced because, at such depolarized potentials, a large fraction of the gates is already in the open position and therefore the sodium current can be generated much faster. However, for the test voltage steps to $-36 \mathrm{mV}$, the delay was increased by $<100 \mu$ s when a $500 \mathrm{~ms}$ conditioning step to $-116 \mathrm{mV}$ instead of the step to $-66 \mathrm{mV}$ preceded the test pulse $(n=3)$ (for the protocol, see Fig. $4 C$ ). Such a small increase in the delay may be attributable to the longer time required to charge the membrane after a large voltage step. In any case, for the test voltage steps to $-36 \mathrm{mV}$, the delay after the $-116 \mathrm{mV}$ conditioning step never exceed $0.4 \times \tau_{m}(n=$ 3 ) and was significantly shorter than the $m^{2}$ function delay of the $0.69 \times \tau_{m}$ (for the formula, see Appendix).

\section{Most of the sodium current kinetic properties are consistent with a single transition model}

The monoexponential activation time course suggests that a single transition controls the kinetics of sodium current activation. If this is the case, for a test step to a given voltage, activation and deactivation time constant of the current should be the same. To test this hypothesis, we analyzed the tail currents obtained with a two-step protocol in the same cell (Fig. $2 F, G$ ).

Voltage steps to $-46 \mathrm{mV}$ that were preceded by a conditioning step to $-36 \mathrm{mV}$ evoked currents with a two-component decay (Fig. $2 F, G)$. We assumed that the slowly decaying component represents the inactivation of the activated current, whereas the rapidly decaying component represents the deactivation process (Oxford, 1981) (for an additional analysis, see below). As shown in Figure $2 \mathrm{H}$, the time constant of the monoexponential fit of the rapidly decaying component was similar to the current activation time constant (Fig. 2E), and, consequently, the traces overlap after normalization.

The close match of activation and deactivation time constants was evident for all of the voltages investigated (Fig. $3 A$ ). Because of technical difficulties, only a limited range between -56 and $-31 \mathrm{mV}$ could be tested. The same close match of activation and deactivation time constants could be detected also at room temperature (Fig. $3 B$ ). The voltage dependence of the time constants remained essentially unaltered at both temperatures: the fits used for the time constants obtained at $12-14^{\circ} \mathrm{C}$ could be used for the time constants measured at $22-24^{\circ} \mathrm{C}$ after division of the fit function by 2.8 , a value close to the expected $Q_{10}$ value (Hille, 2001). These results suggest that no unusual changes in the kinetics of sodium channels occur between $12-14^{\circ} \mathrm{C}$ and $22-24^{\circ} \mathrm{C}$. At room temperature, a direct test of the presence of the delay was less accurate because the first 100-150 $\mu$ s of the recordings after the voltage step were unreliable, and this time is comparable with the time range obtained for the activation time constants (150-400 $\mu \mathrm{s}$ ) (Fig. 3B) (but see below, section about nucleated patch recordings at room temperature in hippocampal CA1 pyramidal neurons and dentate gyrus granule cells). Nevertheless, at room temperature, in prefrontal cortex pyramidal neurons, there was no indication that sodium currents activate after the $m^{n}(n \geq 2)$ type delay.

In a two-state model, the voltage dependence of the current amplitude is usually tied to the voltage dependence of transition rates (Hille, 2001). In prefrontal cortex pyramidal neurons, the activation and deactivation rates increased $e$-fold for each $\sim 12$ $\mathrm{mV}$ (this value tended to increase at membrane potentials below $-60 \mathrm{mV}$ and above $-10 \mathrm{mV}$ ). This voltage dependence of the activation/deactivation rates predicts a value of $6 \mathrm{mV}$ for the slope factor of the activation voltage dependence, which matches the observed slope value (Fig. 3C). For this graph, it was assumed that current decay represents inactivation process, and the total current amplitude was obtained by extrapolation of the decay of the current to the start of the voltage command (for details, see Materials and Methods).

However, it is possible that the actual microscopic time constants do not correspond to the macroscopic time constants (Aldrich et al., 1983; Hille, 2001). For sodium channels, it has been proposed that inactivation process is voltage independent and the apparent voltage dependence of inactivation rate constants is attributable to coupling with activation process (Aldrich et al., 1983; Kuo and Bean, 1994). The following experiments were aimed to test whether a model with a single open state and voltage-independent inactivation can account for the sodium currents behavior in prefrontal cortex pyramidal neurons. 

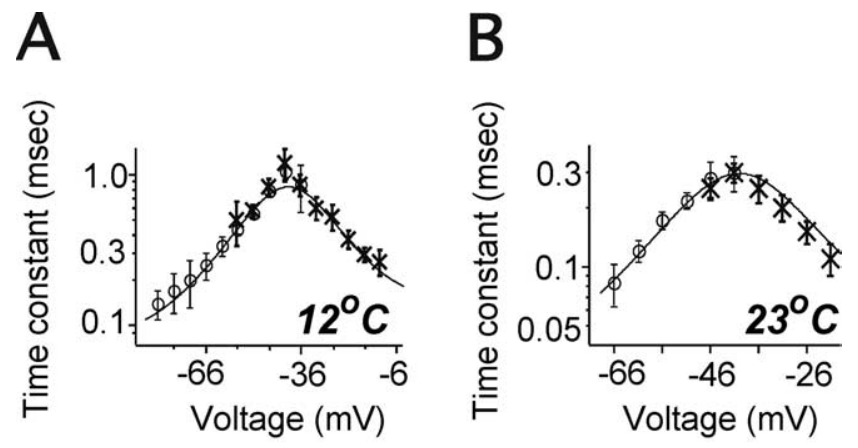

C
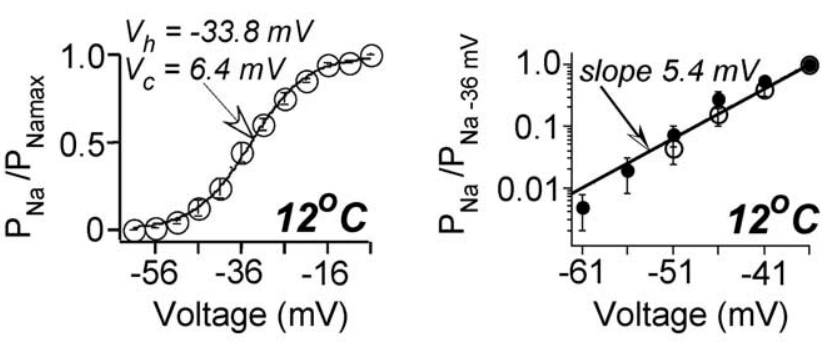

E
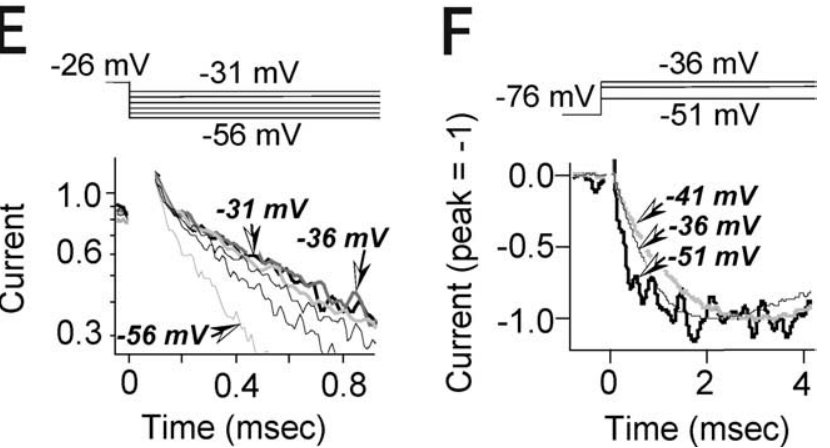

Figure 3. Summary of the properties of sodium current activation in prefrontal cortex pyramidal neurons. $\boldsymbol{A}$, Time constants of activation (crosses) and deactivation (circles) at $12^{\circ} \mathrm{C}$ plotted as a function of the test voltage. All time constants were measured as shown in Figure 2 . The thin line represents the fit function of $1 /(11 /(0.4+\exp (-(V+6) / 12))+0.035 /(0.0015+$ $\exp ((V+6) / 12)))$, where $V$ is the membrane voltage in millivolts. This function was used for computer simulations (for details, see Materials and Methods). $\boldsymbol{B}$, Time constants of activation (circles) and deactivation (crosses) at $23^{\circ} \mathrm{C}$ are plotted as a function of the test voltage. The thin line represents the fit obtained by dividing the fit function for the $12^{\circ} \mathrm{C}$ by 2.8 . C, Peak permeability is plotted as a function of voltage. Permeability was obtained from the corrected peak current amplitudes using the Goldman-Hodgkin-Katz equation. The correction of peak current amplitude was performed by extrapolating the exponential part of the current decay to the start of the test voltage command. Thin line represents a Boltzmann function fit of the form $1 /(1+$ $\exp \left[\left(V_{\mathrm{h}}-V\right) / V_{\mathrm{c}}\right]$, where $V$ is the membrane potential, $V_{\mathrm{h}}$ is the half activation voltage, and $V_{\mathrm{c}}$ is the slope factor. D, Slowly decaying component of tail current (Fig. 2G, open circles) and peak current amplitude (Fig. 1 A, filled circles) are plotted as a function of the test voltage. All data are presented as a relative permeability that was calculated by dividing current amplitude by the instantaneous total tail current amplitude (Fig. $1 \mathrm{~B}$ protocol) at the same voltage. Thereafter, the attained values were normalized to the relative permeability calculated for $-36 \mathrm{mV}$. The amplitude of the slowly decaying component was obtained as shown in Figure 2, $G$ and $H$. The straight line represents the monoexponential fit to the relative permeability values of the slow tail current component. Peak currents were measured during voltage-clamp protocol shown in Figure $1 A$. $E$, Deactivation time constants saturate at approximately $-36 \mathrm{mV}$. The fast component of tail currents was obtained after peeling of the slow component as it is shown in Figure $2 E-H$. The voltage-clamp protocol was the same as in Figures $1 B$ and $2 G$, except that the conditioning step was to $-26 \mathrm{mV}$. To facilitate deactivation rate comparison, tail current amplitudes were set to 1 at the beginning of the test voltage command ( $100 \mu$ s after the end of the conditioning step). The first $100 \mu$ s after the conditioning step to $-26 \mathrm{mV}$ are blanked for clarity. $\boldsymbol{F}$, There is an increase in the sodium current activation rates below $-41 \mathrm{mV}$. Currents were evoked by the voltage-clamp protocol shown at the top. To facilitate activation rate comparison, the amplitude of all currents was set to -1 at the peak of the current.
First, we investigated the voltage dependence of the sodium current at below $-35 \mathrm{mV}$. To this end, in the same neuron, we measured the peak current amplitude (Fig. $1 \mathrm{~A}$ protocol) and divided it by the instantaneous tail current amplitude (Fig. $1 B$ protocol) at the same voltage. If our assumption that the activation process is much faster than the inactivation process is correct, most of the channels are already activated at the peak of the current. Hence, we should obtain relative permeability numbers that should match closely the data obtained in Figure $3 \mathrm{C}$. At membrane voltages above $-51 \mathrm{mV}$, the obtained data (Fig. $3 D$, filled circles) showed similar voltage dependence to the graph in Figure $3 C$. Interestingly, at membrane voltages less than -51 $\mathrm{mV}$, the peak current amplitude seemed to exhibit stronger voltage dependence than at more depolarized membrane potentials.

In addition, we measured the amplitude of the slow tail current component normalized to the instantaneous tail current amplitude at the same voltages. If the fast tail current component represents deactivation process and the slow component represents inactivation process, the normalized slow current amplitude should have voltage dependence similar to the voltage dependence of the peak current. The obtained data (Fig. 3D, open circles) show that both datasets have similar voltage dependence, although some differences could be detected. Notably, the data points corresponding to the slow tail current component were somehow lower than the data points corresponding to the peak current measurements. However, similar behavior could be observed in the computer model described below.

It is important to notice that voltage dependence of both macroscopic activation and deactivation rates had clear peaks (Fig. $3 A)$. Although it was difficult to show a peak of deactivation rates in a single cell (Fig. $3 E$ ), activation rates were clearly the slowest at approximately $-40 \mathrm{mV}$ and started to accelerated at more hyperpolarized membrane potentials as well as at more depolarized potentials (Fig. $3 F$ ). All of these observations will be discussed in the next section while justifying the proposed sodium channel model.

\section{The action potential shape in prefrontal cortex neurons can} be faithfully reproduced with computer simulations

The activation kinetics of sodium currents affects the shape of action potential (Moore and Cox, 1976; Sangrey et al., 2004). Therefore, the validity of the model in which a single transition determines the sodium current activation kinetics can be tested by comparing the shape of action potentials recorded in the prefrontal cortex neurons with the shape of the computer-generated action potentials. To this aim, we developed a computer model that reproduces the obtained current traces during voltage-clamp experiments. Before we discuss the model selection, we present here the data on sodium current inactivation that are necessary for a complete model (Fig. 4).

As shown in Figure 2, $C$ and $D$, at membrane voltages below $-40 \mathrm{mV}$, the current decay could be fit by a monoexponential function with a non-inactivating component, whereas at potentials above $-40 \mathrm{mV}$, an additional slower component was detected. This component, however, accounted for $<30 \%$ of the total current. Similarly, the recovery from inactivation could be usually fit with a monoexponential function. Because we were interested only in the shape of the action potential, we made no attempt to include such a slow current component into the model. Figure $4 B$ shows the summary of the inactivation and recovery from inactivation time constants corresponding to the fast component of each process (Fig. $4 A, B$ ). These time constants peaked at approximately -65 to $-70 \mathrm{mV}$, which was close to the 
half-inactivation voltage (Fig. $4 C, D$ ). These results indicate that a single transition between two states is sufficient to describe the inactivation/deinactivation process.

Based on these results, a six-state model was constructed (Fig. $5 A$ ). Because a complete sodium channel model was not our goal, we selected the simplest model that could reproduce all key observations reported here. First, at the same voltage, macroscopic activation and deactivation rates were similar (Figs. $2 \mathrm{H}, 3 \mathrm{~A}$ ). Second, there was a clear peak of activation rate voltage dependence (Fig. $3 A, F$ ) and good evidence that deactivation rates had a similar peak (Fig. $3 A, E$ ). Third, the total area under the current normalized to the driving force for the sodium ions decreases for voltages more than $-20 \mathrm{mV}$ (data not shown) that contradicts the predictions of a tight coupling between activation and inactivation (Bean, 1981). Moreover, computer simulations (data not shown) suggest that one open-state and multiple closed-state models with voltage independent inactivation (Kuo and Bean, 1994) are unlikely to explain why, for membrane potential change from -41 to $-51 \mathrm{mV}$, the macroscopic activation time constant decreased (Fig. 3F), whereas the macroscopic inactivation time constants increased (Fig. 4B). More sophisticated schemes with several open states (Vandenberg and Bezanilla, 1991) or a modified and more complex version of the Kuo and Bean model (Kuo and Bean, 1994) could explain the observed current behavior; however, we opted for a simpler scheme in which the parameters were more easily related to the observed data.

In our model, the inactivation and activation processes are independent, as it was proposed in the original Hodgkin and Huxley model. We did not use another alternative model that is the one with slow activation and fast voltage-dependent inactivation rates (Hille, 2001). Such a model can well reproduce most of the data presented here (Figs. 1-3). However, we did not see why it should be preferred over an easily interpretable model presented here.

In our model, the activation process was simulated by a twostep process. There were several reasons for the addition of a second closed state. First, there was the $\sim 5$ $\mathrm{mV}$ difference between the half-activation voltage and the voltage at which activation/deactivation time constants reached their peak values (Fig. 3, compare $A, C$ ). Second, at membrane voltages below -60 $\mathrm{mV},>12 \mathrm{mV}$ corresponded to the $e$-fold change in the deactivation time constant (Fig. 3A). Meanwhile, at membrane potentials below $-50 \mathrm{mV}$, the voltage dependence of the current amplitude became steeper (Fig. 3D). This steeper voltage dependence was consistent with the low action potential threshold (Fig. 5D). The addition of a fast voltage-dependent transition between the two closed states was sufficient to account for both observations, although it could not explain all complex voltage dependence of the sodium currents in prefrontal cortex pyramidal neurons (for comparison, see below).

Finally, there was short delay $(\sim 100$ $\mu \mathrm{s})$ of the sodium current activation; imperfect voltage-clamp might account for this delay, but it is also possible that it was
A

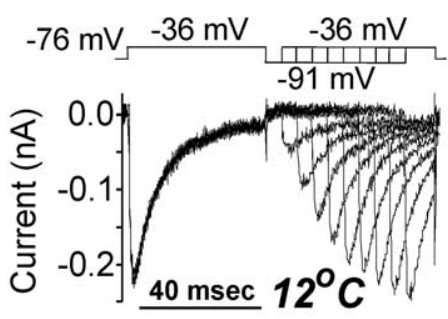

B

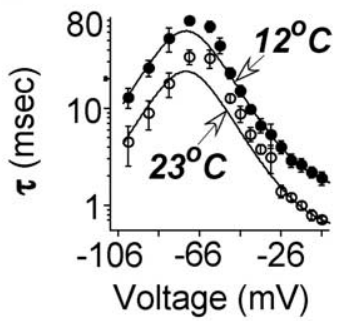

C
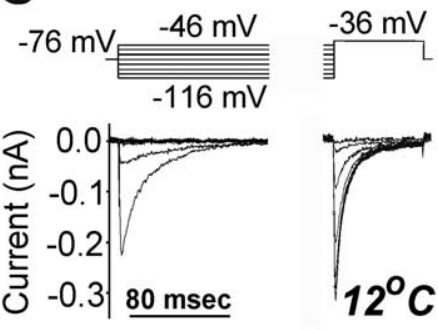

D

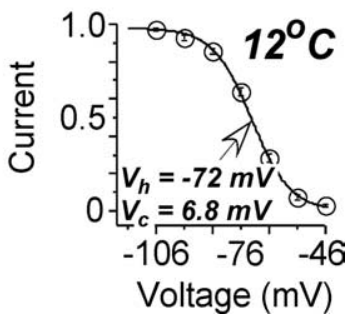

Figure 4. Inactivation properties of sodium currents in prefrontal cortex pyramidal neurons. $\boldsymbol{A}$, Current traces evoked by the voltage protocol used to determine the recovery from inactivation time constants. The protocol is shown above the traces. $\boldsymbol{B}$, Time constants of inactivation and recovery from inactivation plotted as a function of the amplitude of the conditioning voltage step. Inactivation rates were measured by fitting the current decay with the monoexponential or biexponential function. The biexponential fit was required only for voltages above -40 $\mathrm{mV}$. In these cases, the dominant fast component was used for the plot. The thin line for the data points at $12^{\circ} \mathrm{C}$ represents the fit function of $1 /(2 /(2+\exp (-(V+6) / 12))+0.00005 \times$ $\exp (-(V+6) / 13))$, where $V$ is the membrane potential in millivolts. The fit function for the data points at $23^{\circ} \mathrm{C}$ was obtained by dividing the fit function at $12^{\circ} \mathrm{C}$ by 2.4 . The parameters of the fit function were used for the computer simulations (for details, see Materials and Methods). C, Currents evoked by the voltage-clamp protocol shown above the traces. The conditioning step duration was $500 \mathrm{~ms}$. D, Amplitude of the current at $-36 \mathrm{mV}$ plotted as a function of the conditioning step voltage. The thin line represents a Boltzmann function fit.
Figure 5. A two-step activation model accurately reproduces the sodium current behavior and the shape of action potentials in prefrontal cortex pyramidal neurons. $A$, States and transitions scheme used for the computer simulations of the sodium current. C1 and $C 2$ represent the closed states; $11-13$ represent the inactivated states; 0 represents the open state. For functions determining the individual rates, see Materials and Methods. $\boldsymbol{B}$, Simulated currents evoked by a voltage-clamp protocol shown above the traces. The inset shows the start of the activation of the simulated (dark lines) and recorded (light lines) currents for two voltages $(-46$ and $-41 \mathrm{mV})$. The dashed line represents the simulated current response to the $-41 \mathrm{mV}$ voltage step obtained with the $\mathrm{m}^{3}$ model of Mainen et al. (1995). The peak current amplitude was normalized to the peak current amplitude of the model shown in $\boldsymbol{A}$. All traces correspond to the $12^{\circ} \mathrm{C}$. C, Simulated currents were evoked by the voltage-clamp protocol shown above the traces. $D$, In the model, activation (gray circles) and deactivation (gray triangles) rates are similar at the same voltage. Crosses correspond to experimental data from Figure 3 A. Line represents deactivation rates of the $m^{3}$ model $(1 / 3$ of the time constant corresponding to the transition from the closed to the open state in the $\mathrm{HH}$ model). $\boldsymbol{E}$, Activation voltage dependence obtained for simulated currents. Dark thin line represents a Boltzmann function fit. Light thick line corresponds to the experimental data shown in Figure 3C. $\boldsymbol{F}$, In the model, the voltage dependence of the tail current slow component amplitude (open circles) and the peak current amplitude (filled dark circles) are similar. Experimental data of peak current amplitude are shown as gray filled circles. All data are presented as relative permeability obtained by dividing current amplitudes by the instantaneous total tail current amplitude at the start of the test pulse at the same voltage. Thereafter, the attained values were normalized to the relative permeability value obtained at $-26 \mathrm{mV}$. G, $\boldsymbol{H}$, The simulated with the $m^{1}$ sodium channel model action potential (light thick line) is very similar in shape to the action potential recorded experimentally (thin dark line). In $\mathbf{G}$, sodium channels density is the same in soma and axons. In $\boldsymbol{H}$, sodium channels density is approximately eight times higher in axons compared with soma. For current densities used during simulations, see Materials and Methods. For comparison, an action potential simulated with the $m^{3}$ model is shown (dotted line). Note a smoother transition to spike in this model. $I, J$, The membrane potential depolarization speed is reproduced well in the model with the higher sodium channel density in axons. In $I$, sodium channel density is the same in soma and axons. In $J$, sodium channel density is approximately eight times higher in axons compared with soma. Trace notation is the same as in $\boldsymbol{G}$ and $\boldsymbol{H}$. Note clearly asymmetrical time course of the depolarization speed in the recorded spike and in the models with higher axonal channels density. 
A

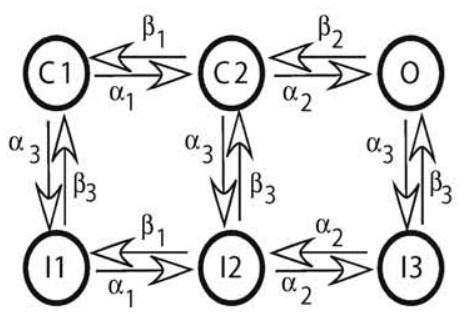

C

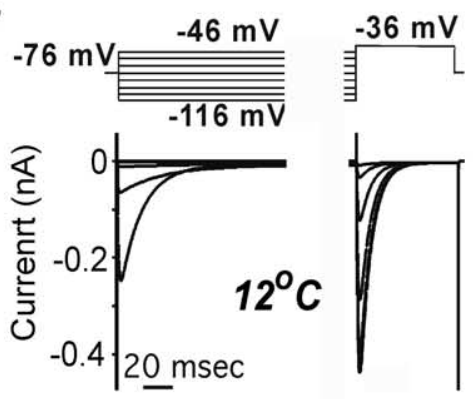

E

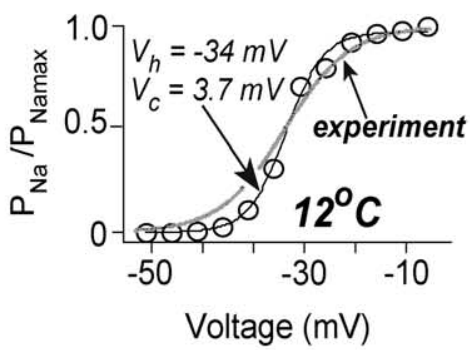

G
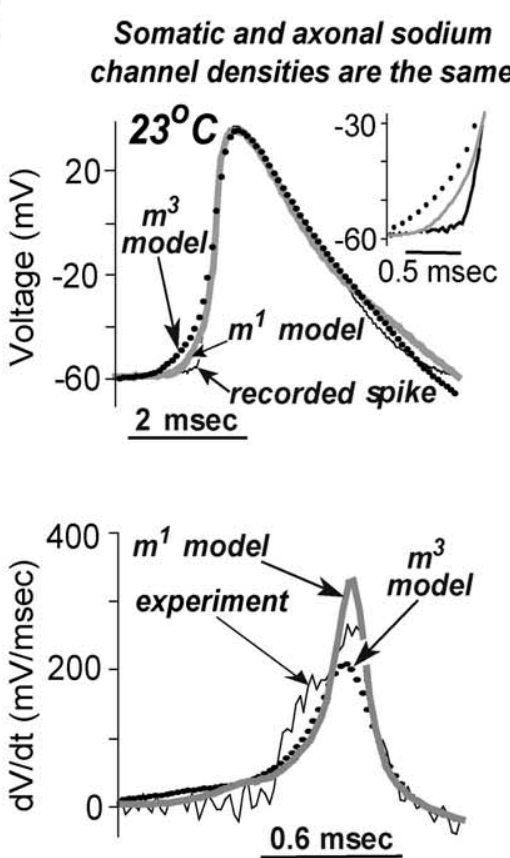

B

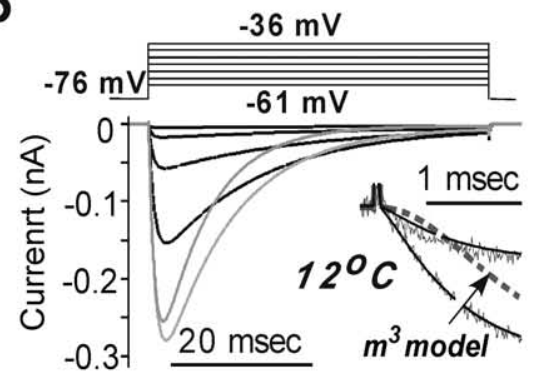

D

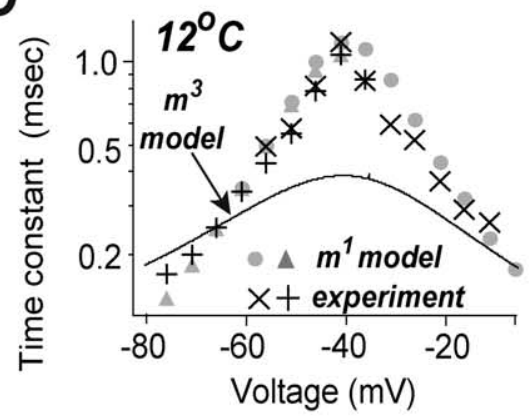

$\mathrm{F}$

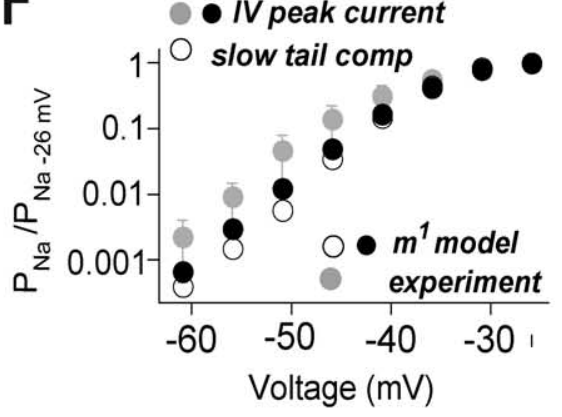

$\mathrm{H}$
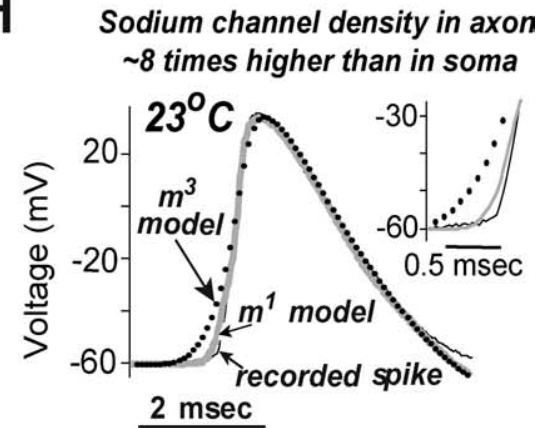

J

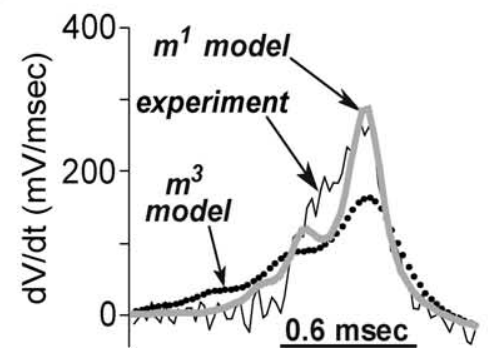

not an experimental artifact (see below) (Lien and Jonas, 2003). Therefore, the peak value of the time constants corresponding to the transition between two closed states was set to be $\sim 100 \mu \mathrm{s}$.

Our model reproduces well both the voltage dependence and kinetics of the sodium currents recorded from prefrontal cortex pyramidal neurons (Fig. $5 B, C$ ). The inset of Figure $5 B$ demonstrates that the time course of sodium current activation matched well the time course of the simulated currents. In contrast, at -41 $\mathrm{mV}$, the amplitude of the current simulated with the $\mathrm{m}^{3}$ kinetics model $[\mathrm{HH}$ model (Mainen et al., 1995)] was approximately threefold smaller during the first $0.5 \mathrm{~ms}$ after the start of the voltage step (Fig. 5B, inset), despite the fact that there is only $\sim 30 \%$ difference between the time constant of the $\mathrm{m}^{3}$ model and the time constant of the model presented here.

For a single trace, it is possible to obtain a much better fit with the HH model; however, in this model, there is a problem of fitting currents at all voltages because voltage dependence of activation and deactivation rates is tied to the voltage dependence of the amplitude of the current. According to the $\mathrm{m}^{3}$ model, the voltage dependence of the amplitude of the current will be $3 \times 2$ times steeper than the voltage dependence of activation and deactivation rates because of the presence of the $m^{3}$ variable. Because the observed voltage dependence of activation and deactivation rates is approximately two times less steep than the observed voltage dependence of the amplitude of the current (Fig. $3 A, C$ ), it is impossible to obtain a satisfactory match of the data reported here with the $m^{3}$ kinetics $\mathrm{HH}$ model. In addition, the $m^{3}$ kinetics HH model cannot explain the similarity of activation and deactivation rates at the same voltage. For all of these reasons, we used the $m^{3}$ kinetics model that was used by several groups without any change in the parameters of the models.

As shown in Figure 5C, all essential inactivation features are reproduced by the model. Although the simulated currents do not contain the more slowly decaying component detected in the recorded currents at potentials above $-41 \mathrm{mV}$ (compare Figs. $1 A, 5 B$ ), this slower component could be added by extending our model, as it has been done for the classical Hodgkin and Huxley model.

One of the key observations reported here is the similarity of activation and deactivation rates at the same voltage over relatively wide range of voltages (from 
-46 to $-31 \mathrm{mV}$ ) (Fig. 3A). Hence, it is crucial that our proposed model can reproduce this phenomenon as shown in Figure $5 D$. Meanwhile, in the model, the same middle point of the voltage dependence of the current amplitude was attained, although there was a large difference in the slope values between the model and the experimental data (3.7 vs $6.4 \mathrm{mV}$ ) (Fig. $5 E$ ). In our model, the increased shift of the half-activation voltage resulted in the decrease of the slope factor. We selected to match the halfactivation voltage while ignoring the slope factor of the Boltzmann function fit. Such choice was dictated by the observation that, for membrane voltages below $-40 \mathrm{mV}$, there was quite good correspondence between this model and the experimental data (Fig. $5 F$ ). In any case, these discrepancies between the model and the experimental data indicate that our model is an oversimplification of the real channel behavior that is not entirely unexpected.

Finally, we tested the models ability to reproduce the shape of action potential. Because the actual sodium channel density in axons is still a matter of debate (Mainen et al., 1995; Safronov et al., 2000), we tested two channel distributions during simulations. First, we assumed that the sodium channel density is the same in the axon and the soma. Under these conditions, a fairly good reproduction of spike shape could be obtained with our model, assuming a sodium channel density within the range of the experimentally observed values. For instance, the observed average sodium channel permeability was $2.5 \times 10^{-4} \mathrm{~cm} / \mathrm{s}$ (ranging from $1.8 \times 10^{-4}$ to $3.9 \times 10^{-4}$ ), whereas for this simulation, a $3.2 \times 10^{-4}$ value was used (Fig. $5 G, I$ ). The main difference between the recorded and the simulated voltage traces is a smoother onset of the spike in the trace of the model (Fig. $5 G$, inset). In the case of the $m^{3} \mathrm{HH}$ model, the simulated spike onset was even slower, although the sodium channel permeability was similar (Fig. 5G) (for the parameters of the model, see Materials and Methods). The steep rising phase of the action potential is attained in the model with approximately eight times higher sodium channel density in the axonal compartment compared with the soma (Fig. $5 \mathrm{H}$, light thick trace). Interestingly, the simulations incorporating the $\mathrm{m}^{3}$ sodium channel model showed little improvement in the steepness of the rising phase (Fig. $5 \mathrm{H}$, dotted trace). This result suggests that our model is more sensitive to local changes in the channels density, probably because of the much faster activation kinetics.

Detailed information on the action potential shape can be obtained by examining the time course of the membrane potential depolarization speed. As shown in Figure 5I, in the case of the same sodium channel density in the soma and the axon, simulations with our model overestimated the maximal depolarization speed, whereas simulations with the $m^{3}$ sodium channel model underestimated the maximal depolarization speed (Fig. $5 F$ ). In addition, both simulations failed to reproduce the asymmetrical profile of the experimentally observed time course of the depolarization speed: the rise to the maximum was slow, whereas the subsequent drop was much faster. However, in the case of high sodium channel density in the axonal compartment, simulations with our model were able to closely reproduce both the time course and the amplitude of the depolarization speed (Fig. 5F, thick light trace), whereas simulation with the $m^{3}$ sodium channel model underestimated approximately two times the peak depolarization speeds. In addition, the initial rising phase of the depolarization speed was very slow compared with the experimental data.

Hence, the presented model is sufficient to reproduce accurately most of the properties of sodium currents and the shape of the action potentials recorded in prefrontal cortex pyramidal neurons. In contrast, the shape of action potentials generated by the model incorporating sodium currents with the $\mathrm{m}^{3}$ kinetics differed from the shape of the experimentally recorded action potential (Fig. 5G-J). In particular, the time course of depolarization speed could be matched only with simulations incorporating our model. We conclude that the presented model can reproduce the observed shape of action potential more faithfully than the traditional Hodgkin and Huxley model.

\section{Sodium current activates with minimal delay in hippocampal CA1 pyramidal neurons and dentate gyrus granule cells}

The described lack of the Hodgkin and Huxley-type delay in the sodium current activation in the prefrontal cortex pyramidal neurons is at odds with the current prevailing view that most, if not all, fast sodium currents activate after the Hodgkin and Huxley-type delay. Therefore, to test whether the sodium current activation time course in prefrontal cortex pyramidal neurons was unique to this cell type or common to other mammalian brain neurons, we recorded sodium currents from two additional types of CNS neurons. We selected hippocampal CA1 pyramidal neurons and dentate gyrus granule cells because they are two well studied and distinct neuron types.

These experiments were performed using the nucleated patch configuration, which provides, probably, the best voltage-clamp conditions (Martina and Jonas, 1997).

In addition, the use of a different experimental approach gave us the opportunity to test whether the observed properties of sodium current activation in the prefrontal cortex neurons were associated with the acute dissociation procedure, which involves protease treatment, the age of animals ( $>4$ weeks for acutely dissociated neurons vs $16-18 \mathrm{~d}$ for nucleated patch recordings), the type of used voltage compensation (hypercharge in acutely dissociated cells vs traditional exponential charging), or the specific solution composition used to record sodium currents (for details, see Materials and Methods). Finally, the $-124 \mathrm{mV}$ prepulse was used during nucleated patch recordings to boost the recorded current amplitude. At $-120 \mathrm{mV}$, we expected all sodium channels to be closed, preventing any possible complications associated with the relatively depolarized holding potential used in the previous experiments.

All experiments on nucleated patches were performed at room temperature $\left(21-23^{\circ} \mathrm{C}\right)$.

Despite the above-mentioned differences in the experimental details, the properties of the sodium current activation in the hippocampal CA1 pyramidal neurons and dentate gyrus granule cells were qualitatively the same as in the prefrontal cortex pyramidal neurons. Figure $6 \mathrm{~A}$ shows a typical set of traces obtained from an hippocampal CA1 neuron. Looking at the expanded time scale traces shown in the Figure $6 B$, at first glance, one might have the impression that sodium currents activate after a relatively long delay. However, because of the extreme speed of the sodium current activation, first we need to establish experimentally how quickly the voltage clamp was established after the voltage step.

Most commercial amplifiers, including the Axopatch 200B used for these experiments, in addition to the devices that speed up membrane charging at the beginning of the voltage command ("active compensation" according to the Axopatch 200B manual), have a circuitry that subtracts from the recorded trace the membrane charging transient current ("passive compensation"). Hence, the absence of any charging current in the traces does not mean that the membrane charging process has been completed 
A

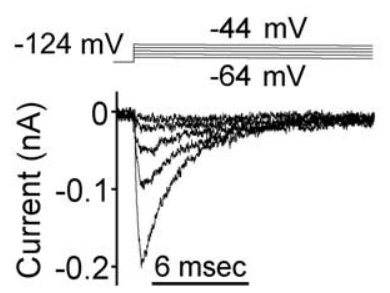

C

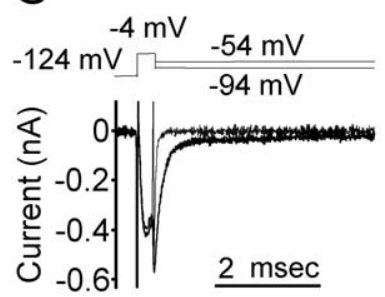

$\mathrm{E}$

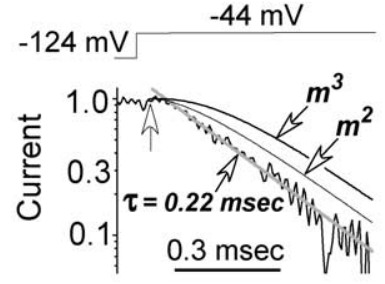

G

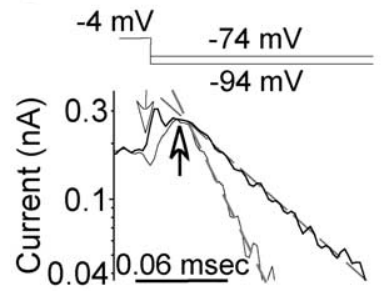

B
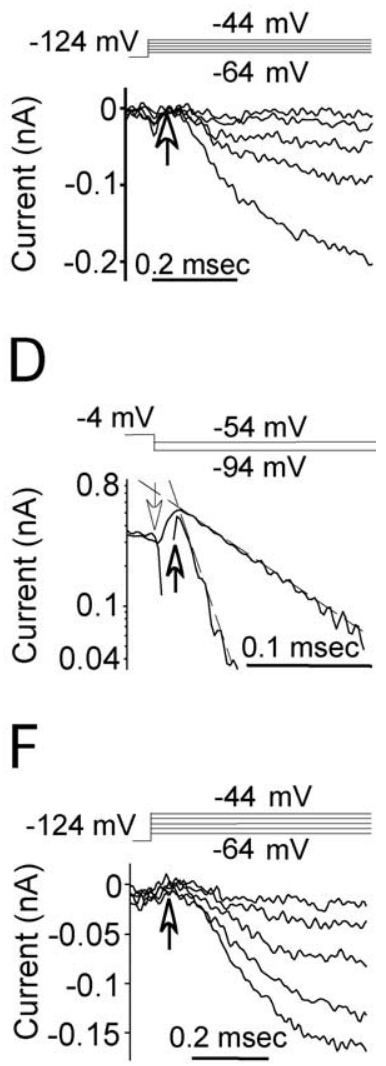

$\mathrm{H}$

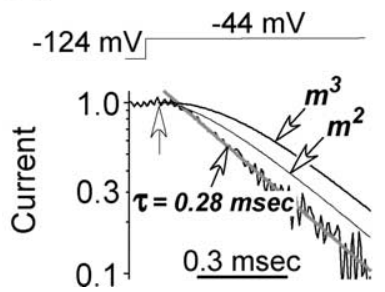

Figure 6. Sodium currents activated with minimal delay in hippocampal CA1 pyramidal neurons $(\boldsymbol{A}-\boldsymbol{E})$ and the dentate gyrus granule cells $(\boldsymbol{F}-\boldsymbol{H})$ at $22^{\circ} \mathrm{C}$. $\boldsymbol{A}, \boldsymbol{B}$, Sodium current activation recorded in a nucleated patch obtained from a hippocampal CA1 pyramidal neuron. Currents were evoked by the voltage-clamp protocol shown above the traces. In $\boldsymbol{B}$, traces are shown on expanded time scale. An arrow indicates an estimated time point when the membrane charging was completed by $\sim 80 \%$ as estimated from tail current traces shown in $\boldsymbol{D}$. C, D, Tail currents evoked by the protocol shown above the traces. In $\boldsymbol{D}$, traces are shown in the expanded semilogarithmic plot. The light arrow indicates the onset of the voltage step command, and the dark arrow indicates the time point when current traces differed $<20 \%$ from the monoexponential fit of the linear part of the trace in the semilogarithmic plot. $E$, A semilogarithmic plot of the modified current trace that was obtained from $\boldsymbol{A}$ following the procedure shown in Figure 2. This procedure included two steps of modifications. First, inactivation of the current was eliminated mathematically by dividing the current trace by the following function: $17.8 \mathrm{pA}+227.4$ $\mathrm{pA} \times \exp (-t / 2.22 \mathrm{~ms})$. Second, the final trace was obtained after the subtraction of the obtained trace from 1. For details of all procedures, see Appendix. The thick light line represents the monoexponential fit. The two smooth lines correspond to the quadrate (thin line) and the cube (thick line) of an exponential function. The light arrow indicates the time point when the membrane charging was completed by $\sim 80 \%$ as estimated from tail current traces. $\boldsymbol{E}$, Sodium current activation recorded in a nucleated patch obtained from a dentate gyrus granule cell. $\boldsymbol{F}$, Tail currents plotted in the semilogarithmic plot were evoked by the protocol shown above the traces. The light arrow indicates the onset of the voltage step command, and the dark arrow indicates the time point when current traces differed $<20 \%$ from the monoexponential fit of the linear part of the trace in the semilogarithmic plot. $\boldsymbol{H}$, A semilogarithmic plot of the modified current trace that was obtained from $\boldsymbol{F}$ following the procedure shown in Figure 2 . All notations are the same as in $\boldsymbol{E}$.

and the applied voltage is sensed by the membrane. Because we applied test voltages of -64 to $-44 \mathrm{mV}$ from the holding potential of $-124 \mathrm{mV}$, a $20 \%$ reduction in the voltage command would result in a voltage difference of $\sim 10 \mathrm{mV}$. Figure $6 \mathrm{~A}$ shows that, for the command step from -124 to $-44 \mathrm{mV}$, no current would be detected if the membrane sensed only $80 \%$ of the $80 \mathrm{mV}$ voltage difference, which translates into a membrane potential of $-44 \mathrm{mV}-0.2 \times 80 \mathrm{mV}=-60 \mathrm{mV}$. Therefore, we need to know the time required to achieve $\sim 80 \%$ of the voltage command step amplitude on the cell membrane. This time can be estimated from the tail currents by identifying the time point at which the current trace deviates $>20 \%$ from the monoexponential function.

The amplitude of the voltage steps used to evoke tail currents was similar to the amplitude of the voltage steps used for the delay measurements (Fig. 6A,B). Figure $6 D$ shows tail current traces on an expanded semilogarithmic scale. It is clear that $>30 \mu$ s was required to charge the membrane, because no tail currents were detectable during the first $30 \mu \mathrm{s}$. The arrow indicates the time point corresponding to the average membrane charging duration that was sufficient to ensure that the deviation of the current trace from the monoexponential time course was $<20 \%$. This time point was $\sim 35 \mu$ s after the onset of the voltage step command. In this recording, the estimated series resistance was $10 \mathrm{M} \Omega$, and the patch capacitance that corresponds to the patch area of $\sim 70 \mu \mathrm{m}^{2}$ was $1 \mathrm{pF}$. Hence, for uncompensated series resistance, the calculated time constant would be $\sim 10 \mu$ s. This estimate shows that other factors, such as digital filter time constant $(\sim 10 \mu$ s of the 20 $\mathrm{kHz}$ low-pass filter), command lag ( $\sim 5 \mu \mathrm{s})$, compensation circuitry lag $(\sim 15 \mu \mathrm{s})$, and pipette stray capacitance, have contributed to a relatively slow onset of the tail current.

The arrow in the Figure $6 B$ indicates the time point $35 \mu$ s after the start of the voltage step. Now the detected delay is approximately two times shorter than one would measure without the correction derived from the tail current analysis. Following the procedure described in Figure 2, a direct comparison of the observed delay and the expected delay can be performed (Fig. $6 E$ ). The traces corresponding to the $\mathrm{m}^{2}$ and $\mathrm{m}^{3}$ functions were shifted to the right by $35 \mu$ s. There are no obvious differences between Figures $2 E$ and $6 E$, except for much faster activation time constant at $22^{\circ} \mathrm{C}$. In hippocampal CA1 pyramidal neurons, for voltage steps to $-44 \mathrm{mV}$, the average delay was $71 \pm 13 \mu$ s or $(0.16 \pm 0.04) \times \tau_{m}(n=4)$. Similarly, in dentate gyrus granule cells, for voltage steps to $-44 \mathrm{mV}$, the observed average delay was $50 \pm 5 \mu$ s or $(0.18 \pm 0.02) \times \tau_{m}(n=4)$ (Fig. $\left.6 F-H\right)$.

Interestingly, the average delay changed little with voltage. In hippocampal CA1 pyramidal neurons, the delay was $71 \mu$ s at -44 $\mathrm{mV}$ and $69 \mu \mathrm{s}$ at $-29 \mathrm{mV}(n=4)$. Meanwhile, in the same neurons, the activation time constant differed $\sim 1.7$ times for these two membrane potentials ( $396 \mu$ s at $-44 \mathrm{mV}$ and $237 \mu$ s at $-29 \mathrm{mV} ; n=4)$. This relative insensitivity to the membrane voltage in this range of membrane potentials is inconsistent with the Hodgkin and Huxley model, which predicts a linear relationship between the delay and the activation time constant. Nevertheless, there was a clear decrease in the delay for voltage steps to less than $-45 \mathrm{mV}$. For voltage steps to $-49 \mathrm{mV}$, the delay duration observed in the currents from the hippocampal CA1 pyramidal neurons was $41 \mu \mathrm{s}(n=3)$. Although the small current amplitude complicated the analysis at these voltages, the observed trend is consistent with the proposed model of sodium current activation. 


\section{Discussion}

Analysis of the sodium current gating in three different types of central neurons showed that, in all of them, sodium currents activated without a Hodgkin and Huxley-type delay. A brief delay that could not be attributed to the experimental artifact was two to five times shorter than the delay predicted by the Hodgkin and Huxley model. In addition, at the same voltage, the activation and deactivation time constants were indistinguishable. These results are consistent with a model in which a single transition from the closed to the open state determines the activation kinetics of sodium currents. Computer simulations with such a sodium current model reproduced well the shape of the action potential recorded in the prefrontal cortex neurons. In contrast, computer simulations with the traditional Hodgkin and Huxley-type model failed to reproduce the sharp rising phase of the experimentally recorded action potentials (Fig. 5D).

Our results show that the usual assumption that, in the majority of neurons, sodium currents activate after the Hodgkin and Huxley-type delay (Traub and Milles, 1991; Mainen et al., 1995) is probably incorrect. Over the membrane voltage range tested in this paper, the observed brief delay that cannot be attributed to experimental artifacts is primarily voltage independent and too brief to be fit with the $m^{n}$ function. For membrane voltages between -55 and $-25 \mathrm{mV}$, the observed delay was at least two to five times shorter than is predicted by the $m^{2}$ function and at least three to seven times shorter than is predicted by the $\mathrm{m}^{3}$ function.

The gating model presented here, conversely, did reproduce that brief delay (Fig. $5 B$ ) by adding a transition between two closed states. For membrane voltages from -45 to $-30 \mathrm{mV}$, this transition was assumed to be much faster (more than approximately five times) than the second transition from the closed to the open state, which determines the activation/deactivation time constants of the currents (for details, see Materials and Methods). In this range of membrane voltages, the approximately fivefold difference in transition rates corresponds to the observed delay duration, ranging from $0.13 \times \tau_{m}$ to $0.25 \times \tau_{m}$, where $\tau_{m}$ is the activation time constant. Comparison of simulated current traces with the experimentally recorded currents (Figs. 1, 2, 5) suggest that our model reproduces well most of the sodium current activation properties observed in prefrontal cortex pyramidal neurons.

In addition, we tested the validity of our model by reproducing current-clamp data obtained from the prefrontal cortex pyramidal neurons. Although many currents shape neuronal responses, the action potential shape is very sensitive to changes in the sodium current activation time course (Moore and Cox, 1976; Clay, 1998; Sangrey et al., 2004). Therefore, we reasoned that a good model should be able to reproduce spike shape with current densities that are close to the experimentally observed values. For comparison, we selected the traditional $\mathrm{m}^{3}$ sodium channel model (Mainen et al., 1995) that has been used by several groups. As shown in Figure $5 G-J$, the steep rising phase of the recorded action potential was reproduced significantly better during simulations with our model. In addition, during simulations with our model, we were able to attain a close match between the simulated and experimental time course and amplitude of the membrane potential depolarization speed.

It is rather surprising that nobody attempted to carefully analyze the shape of the sodium currents activation time course in central mammalian neurons. Although several papers reported data on sodium current activation and deactivation time constants in central neurons (for example, Mainen et al., 1995; Mar- tina and Jonas, 1997), none of them attempted to identify the shape of the activation time course of the current as it was done for the sodium currents recorded in the large peripheral axons (Goldman and Schauf, 1973; Keynes and Rojas, 1976; Neumcke et al., 1976; Neumcke and Stampfli, 1982). This fact could have several explanations. First, it seems likely that researchers assumed that such an analysis is impossible because sodium currents activate extremely rapidly. A second possibility is that, with most commercial amplifiers, the quality of the achieved voltage clamp may be deceiving, as is demonstrated in Figure 6. Moreover, we discovered that, in some amplifiers such as Axopatch $200 \mathrm{~B}$, the $20 \mathrm{kHz}$ setting of the low-pass filter that was necessary for the analysis of our experiments is absent and a software-based signal filtering was used (see Materials and Methods).

Finally, we cannot exclude that, outside of the tested here membrane potential range (above $-25 \mathrm{mV}$ ), the activation time course can be fitted with the $m^{n}$ function. We focused on the range of membrane voltages at which sodium current activation is the slowest (from -55 to $-25 \mathrm{mV}$ ), and the duration of the delay is clearly smaller than it is predicted by the $m^{n}$ function. Extrapolation of our data to more depolarized potentials suggest that, at $\sim 0 \mathrm{mV}$, the activation time course may be fit with the $\mathrm{m}^{2}$ function. Nevertheless, this current behavior is primarily reproduced by the computer model because, for the membrane potentials $\geq 0 \mathrm{mV}$, the two transition rates that describe the channels opening differ less than two times, and the delay duration introduced by our model approaches the delay duration predicted by the $m^{2}$ function (see Materials and Methods). Hence, the conclusions drawn from our computer simulations are not affected by the relatively longer delays at more depolarized potentials.

Besides the differences in the predicted action potential shape and the effects of sodium channel distribution, there are two additional important consequences of our observations.

First, our data show that, in sodium channels of central mammalian neurons, a single gate controls most of the channel kinetics. Such a conclusion is based on a number of observations, including the similarity between activation and deactivation time constants (Fig. 3A), the presence of the minimum in the voltage dependence of activation and deactivation time constants (Fig. $3 E, F)$, and good correspondence between voltage dependence of activation kinetics and current amplitudes (Fig. $3 A-D$ ). Hence, it is likely that a unique structural motif is responsible for most of the kinetics governing the opening of sodium channels. The identification of this structural motif will require experiments in heterologous expression systems. Interestingly, there is no detailed study on sodium current activation time course for currents recorded in heterologous expression systems. Thus, our data suggest a new venue for the structure-function studies in sodium channels.

Second, it is known that the amplitude of current fluctuations depends on the current activation time course (Steinmetz et al., 2000). For the same channel density, the fluctuation amplitude is larger when the current activation time course is described by a monoexponential function than when it is described by the cube of an exponential function. Because membrane potential fluctuations modulate coincidence detection, it is possible that the absence of the HH-type delay of the sodium current activation will be reflected in the coincidence detection (Wiesenfeld and Moss, 1995). In other words, information processing in neurons may be influenced by the activation time course of sodium currents. However, a detailed study is required to support this hypothesis.

In conclusion, sodium currents in three types of central neurons activate with a brief delay, the duration of which is much 
smaller than predicted by the Hodgkin and Huxley-type model. The data presented here change our understanding of how sodium currents determine the shape of action potentials and, consequently, influence calcium entrance during the spike, a crucial process for brain plasticity and memory (Deisseroth et al., 2003).

\section{Appendix}

A modified procedure used by Keynes and Rojas (1976) was used to directly determine the activation time constant $\tau_{m}$ and $n$ in the $\left[1-\exp \left(-t / \tau_{\mathrm{m}}\right)\right]^{n}$ function (see below).

According to the Hodgkin-Huxley model, the shape of sodium currents activated by a voltage step from hyperpolarized membrane potentials can be described by the following equation (Hodgkin and Huxley 1952, their Eq. 19): $g_{\mathrm{Na}}=g_{\mathrm{Namax}} \times[1-$ $\left.\exp \left(-t / \tau_{m}\right)\right]^{3} \times \exp \left(-t / \tau_{\mathrm{h}}\right)$, where $g_{\mathrm{Na}}$ is the amplitude of the sodium current, $\mathrm{g}_{\mathrm{Namax}}$ is the maximal amplitude of the sodium current at the test potential, $t$ is the time from the start of the voltage step, $\tau_{m}$ is the activation time constant at the test voltage, and $t_{h}$ is the inactivation time constant at the test voltage. A more generalized version of this function is the following: $g_{\mathrm{Na}}=g_{\mathrm{Namax}}$ $\times\left[1-\exp \left(-t / \tau_{m}\right)\right]^{n} \times \exp \left(-t / \tau_{h}\right)$.

Because is usually set to be equal to 2 or 3 , this general function describing sodium current activation is here referred to as the $m^{n}$ function. The procedure consisted of the following steps.

First, the TTX-sensitive currents were corrected for inactivation by dividing by $g_{\text {Namax }} \times \exp \left(-t / \tau_{h}\right)+g_{\text {non-inac }}$, in which $g_{\text {non-inac }}$ represents the non-inactivating term of the monoexponential fit function or the non-inactivating plus slowly inactivating terms of the biexponential fit function. The fit was performed in a semilogarithmic plot (Fig. 2G) using peeling procedure for the non-inactivating and slowly inactivating components, if they were present.

Following such a procedure, in the case of the $m^{3}$ kinetics, the obtained current shape can be described by the following function: $I_{1}=\left[1-\exp \left(-t / \tau_{m}\right)\right]^{3}$.

Second, the normalized current (Fig. 2D, inset) was subtracted from 1 . In the case of the $m^{3}$ kinetics, following this procedure, we obtain the following function: $I_{2}=1-[1-\exp (-t /$ $\left.\left.\tau_{m}\right)\right]^{3}=1-1+3 \times \exp \left(-t / \tau_{m}\right)-3 \times\left[\exp \left(-t / \tau_{m}\right)\right]^{2}-$ $\left[\exp \left(-t / \tau_{m}\right)\right]^{3}$ and $I_{2}=3 \times \exp \left(-t / \tau_{m}\right)-3 \times\left[\exp \left(-t / \tau_{m}\right)\right]^{2}-$ $\left[\exp \left(-t / \tau_{m}\right)\right]^{3}$.

Because $\left[\exp \left(-t / \tau_{m}\right)\right]^{2}$ and $\left[\exp \left(-t / \tau_{m}\right)\right]^{3}$ decay faster than $\exp \left(-t / \tau_{m}\right)$, for $t \gg \tau_{m}, I_{2} \sim 3 \times \exp \left(-t / \tau_{m}\right)$.

In the case of the $m^{2}$ kinetics, this procedure produces the following function: $I_{2}=2 \times \exp \left(-t / \tau_{m}\right)-\left[\exp \left(-t / \tau_{m}\right)\right]_{2}$.

For $t \gg \tau_{m}$, it becomes the following: $I_{2} \sim 2 \times \exp \left(-t / \tau_{m}\right)$.

In the case of the $m^{1}$ kinetics, we obtain the following function: $I_{2}=\exp \left(-t / \tau_{m}\right)$. Therefore, in a semilogarithmic plot, for $t$ $\gg \tau_{m}$, the obtained current trace will approach the line corresponding to the slope of $\exp \left(-t / \tau_{m}\right)$ (Fig. $2 E$ ). The only difference between the graphs corresponding to the $\mathrm{m}^{3}, \mathrm{~m}^{2}$, and $\mathrm{m}^{1}$ kinetics will be a delay that corresponds to the coefficient in front of the exponential term, which is $\ln (3) \times \tau_{m}$ in the case of the $m^{3}$ kinetics, $\ln (2) \times \tau_{m}$ in the case of the $m^{2}$ kinetics, and no delay in the case of the $m^{1}$ kinetics.

Hence, this method permits a relatively independent way to estimate $\tau_{m}$ and $n$ in the $m^{n}$ function. In addition, this procedure provides an estimate of the maximal time allowed to attain the desired voltage-clamp control after the voltage step so as not to impair the identification of the $n$ in the $m^{n}$ function. For example, if we want to discriminate between the $m^{2}$ and $m^{1}$ kinetics, we have to detect the $\ln (2) \times \tau_{m}$ delay of $I_{2}$. Because $\tau_{m}$ is $\leq 0.3 \mathrm{~ms}$ at $23^{\circ} \mathrm{C}$ (Fig. 3B) (Martina and Jonas, 1997), the membrane poten- tial should stabilize in $<0.3 \times \ln (2)=0.2 \mathrm{~ms}$. During the experiments described here, the estimated membrane potential stabilization times (Fig. 1) were $\sim 0.1-0.15 \mathrm{~ms}$, which is insufficient in most of the recordings at room temperatures. However, at $13^{\circ} \mathrm{C}$, $\tau_{m}$ can be $>0.5 \mathrm{~ms}$ (Fig. $3 A$ ), and the $0.15 \mathrm{~ms}$ membrane charging time allows the discrimination between the $m^{2}$ and $m^{1}$ kinetics.

In the majority of studies using whole-cell recordings, no experimental data are provided to estimate the duration of membrane potential stabilization after the voltage step. Without direct evidence, it is difficult to estimate the time required to establish the desired voltage-clamp control after a voltage command. A typical estimate based on the current transient in response to a voltage step does not provide a good estimate of stabilization time for most commercial amplifiers (Sherman et al., 1999). The estimated response time of the Axoptach 1D amplifier was always $>90 \mu$ s (Sherman et al., 1999), and it is probably safe to say that it takes $\sim 0.1-0.2 \mathrm{~ms}$ to attain a sufficient voltage-clamp control after a voltage step. Therefore, during whole-cell recordings at room temperature, it is nearly impossible to detect the $m^{2}$-type delay of sodium currents.

\section{References}

Aldrich RW, Corey DP, Stevens CF (1983) A reinterpretation of mammalian sodium channel gating based on single channel recording. Nature 306:436-441.

Armstrong CM, Chow RH (1987) Supercharging: a method for improving patch-clamp performance. Biophys J 52:133-136.

Baranauskas G (2004) Cell-type-specific splicing of KChIP4 mRNA correlates with slower kinetics of A-type current. Eur J Neurosci 20:385-391.

Bean BP (1981) Sodium channel inactivation in the crayfish giant axon. Must channels open before inactivating? Biophys J 35:595-614.

Catterall WA (2000) From ionic currents to molecular mechanisms: the structure and function of voltage-gated sodium channels. Neuron 26:13-25.

Clay JR (1998) Excitability of the squid giant axon revisited. J Neurophysiol 80:903-913.

Deisseroth K, Mermelstein PG, Xia H, Tsien RW zens(2003) Signaling from synapse to nucleus: the logic behind the mechanisms. Curr Opin Neurobiol 13:354-365.

Durstewitz D, Seamans JK, Sejnowski TJ (2000) Dopamine-mediated stabilization of delay-period activity in a network model of prefrontal cortex. J Neurophysiol 83:1733-1750.

French RJ, Horn R (1983) Sodium channel gating: models, mimics, and modifiers. Annu Rev Biophys Bioeng 12:319-356.

Goldman L, Schauf CL (1973) Quantitative description of sodium and potassium currents and computed action potentials in Myxicola giant axons. J Gen Physiol 61:361-384.

Groenewegen HJ, Uylings HB (2000) The prefrontal cortex and the integration of sensory, limbic and autonomic information. Prog Brain Res 126:3-28.

Hille B (2001) Ion channels of excitable membranes. Sunderland, MA: Sinauer.

Hines M (1993) Neuron-a program for simulation of nerve equations. In: Neural systems: analysis and modeling (Eeckman FH, ed), pp 127-136. Boston: Kluwer.

Hodgkin AL, Huxley AF (1952) A quantitative description of membrane currents and its application to conductance and excitation in nerve. J Physiol (Lond) 117:500-544.

Huguenard JR, Hamill OP, Prince DA (1989) Sodium channels in dendrites of rat cortical pyramidal neurons. Proc Natl Acad Sci USA 86:2473-2477.

Kawaguchi Y (1993) Groupings of nonpyramidal and pyramidal cells with specific physiological and morphological characteristics in rat frontal cortex. J Neurophysiol 69:416-431.

Keynes RD, Rojas E (1976) The temporal and steady-state relationships be tween activation of the sodium conductance and movement of the gating particles in the squid giant axon. J Physiol (Lond) 255:157-189.

Korngreen A, Sakmann B (2000) Voltage-gated $\mathrm{K}^{+}$channels in layer 5 neocortical pyramidal neurones from young rats: subtypes and gradients. J Physiol (Lond) 525:621-639.

Kuo CC, Bean BP (1994) $\mathrm{Na}^{+}$channels must deactivate to recover from inactivation. Neuron 12:819-829. 
Lien CC, Jonas P (2003) Kv3 potassium conductance is necessary and kinetically optimized for high-frequency action potential generation in hippocampal interneurons. J Neurosci 23:2058-2068.

Mainen ZF, Joerges J, Huguenard JR, Sejnowski TJ (1995) A model of spike initiation in neocortical pyramidal neurons. Neuron 15:1427-1439.

Martina M, Jonas P (1997) Functional differences in $\mathrm{Na}^{+}$channel gating between fast-spiking interneurones and principal neurones of rat hippocampus. J Physiol (Lond) 505:593-603.

Maurice N, Tkatch T, Meisler M, Sprunger LK, Surmeier DJ (2001) $\mathrm{D}_{1} / \mathrm{D}_{5}$ dopamine receptor activation differentially modulates rapidly inactivating and persistent sodium currents in prefrontal cortex pyramidal neurons. J Neurosci 21:2268-2277.

Migliore M, Messineo L, Cardaci M, Ayala GF (2001) Quantitative modeling of perception and production of time intervals. J Neurophysiol 86:2754-2760.

Moore JW, Cox EB (1976) A kinetic model for the sodium conductance system in squid axon. Biophys J 16:171-192.

Neher E (1992) Correction for liquid junction potential in patch clamp experiments. Methods Enzymol 207:123-131.

Neumcke B, Stampfli R (1982) Sodium currents and sodium-current fluctuations in rat myelinated nerve fibres. J Physiol (Lond) 329:163-184.

Neumcke B, Nonner W, Stampfli R (1976) Asymmetrical displacement current and its relation with the activation of sodium current in the membrane of frog myelinated nerve. Pflügers Arch 363:193-203.

Oxford GS (1981) Some kinetic and steady state properties of sodium channels after removal of inactivation. J Gen Physiol 77:1-22.

Patlak J (1991) Molecular kinetics of voltage-dependent $\mathrm{Na}^{+}$channels. Physiol Rev 71:1047-1080.
Safronov BV, Wolff M, Vogel W (2000) Excitability of the soma in central nervous system neurons. Biophys J 78:2998-3010.

Sangrey TD, Friesen WO, Levy WB (2004) Analysis of the optimal channel density of the squid giant axon using a reparameterized Hodgkin-Huxley model. J Neurophysiol 91:2541-2550.

Sherman AJ, Shrier A, Cooper E (1999) Series resistance compensation for whole-cell patch-clamp studies using a membrane state estimator. Biophys J 77:2590-2601.

Steinmetz PN, Manwani A, Koch C, London M, Segev I (2000) Subthreshold voltage noise due to channel fluctuations in active neuronal membranes. J Comput Neurosci 9:133-148.

Stuart GJ, Sakmann B (1994) Active propagation of somatic action potentials into neocortical pyramidal cell dendrites. Nature 367:69-72.

Tkatch T, Baranauskas G, Surmeier DJ (1998) Basal forebrain neurons adjacent to the globus pallidus co-express GABAergic and cholinergic marker mRNAs. NeuroReport 9:1935-1939.

Traub RD, Milles R (1991) Neuronal networks of the hippocampus. Cambridge, UK: Cambridge UP.

Vandenberg CA, Bezanilla F (1991) A sodium channel gating model based on single channel, macroscopic ionic, and gating currents in the squid giant axon. Biophys J 60:1511-1533.

Vysokanov A, Flores-Hernandez J (1998) mRNAs for clozapine-sensitive receptors co-localize in rat prefrontal cortex neurons. Neurosci Lett 258:179-182.

Wiesenfeld K, Moss F (1995) Stochastic resonance and the benefits of noise: from ice ages to crayfish and SQUIDs. Nature 373:33-36. 